\title{
Normprobleme bei der Pluralbildung fremder und nativer Substantive
}

\author{
Heide Wegener (Potsdam)
}

\begin{abstract}
The paper takes the German noun plural formation as an example for different cases of doubt and shows in which circumstances German speakers can have problems to create the "normal" plural form corresponding to the standard. The paper distinguishes between native and non native plural forms. The cases of doubt within the former can be shown to result from either natural change which leads to a reduction of plural classes and explains the decline of the erand the umlaut plural, or from a strategy of compensation which replaces the non iconic 0plural by forms in $-n$ or $-s$. The problems with the non native nouns varying between a plural form in $-s$ and one ending in a schwa suffix are shown to be the consequence of the ongoing assimilation process. The $-s$ is in complementary distribution with the native schwa suffixes of German and a means of integration: The non-syllabic $-s$ allows for highly corresponding, "conservative" forms similar to the singular by adding only a segment, not a syllable to the stem. By contrast, a native plural with a syllabic suffix alters more or less severely the phonological shape of the base. The alterations yield a continuum of similarity that are demonstrated in a diagram. The paper shows that this distribution is functionally motivated. Loan words, nouns not yet fully established in the speech community, can only be altered in their phonological structure after they have gained a certain degree of familiarity. Only the established borrowings apply - en or - $e$ because these allow "better" plural forms as to prosodic and phonological constraints, i.e. trochaic forms without consonant clusters and superheavy syllables. Finally, the paper discusses the consequences of this variation for the layers of the lexicon and for the grammar of German. Three appendixes indicate the statistical development of the plural classes during the last century, token frequencies of some examples and regional differences in the use of the variants.
\end{abstract}

\section{$1 \quad$ Fragestellungen}

Normprobleme und damit Zweifelsfälle hinsichtlich der Pluralbildung bestehen im derzeitigen Deutsch bei nativen und fremden Substantiven in mindestens neun unterscheidbaren Fällen. Die Gemeinsamkeiten der betroffenen Nomen erlauben es, hier von Klassen von Zweifelsfällen zu sprechen. 
Für jede dieser Zweifelsfallklassen sollen die Ursachen für die Entstehung der Varianten erklärt und wenn möglich diejenige Variante bestimmt werden, für die die größte Wahrscheinlichkeit besteht, dass sie sich durchsetzen wird. Der Beitrag wird die verschiedenen Fälle durch einschlägige Beispiele exemplifizieren und ihre Entstehung erklären, er thematisiert zum Schluss zwei theoretische Fragen: 1. inwiefern Zweifelsfälle eine natürliche Erscheinung der Sprache bzw. der Sprachentwicklung und also unvermeidlich sind, 2. welche Bedeutung die hier beobachteten Fälle für die Grammatik der deutschen Sprache haben.

\section{Zweifelsfälle bei nativen Pluralformen}

\subsection{Abbau von Markiertheit}

Bei nativen Pluralformen entsteht Variation durch Abbau von derzeit zwei kleinen, irregulären Klassen, und zwar des er-Plurals und des Umlautplurals (eigentlich - $(e)+$ Umlautplural).

Im ersteren Fall sind Maskulina und Neutra betroffen, deren Pluralbildung zwischen Formen auf - er und solchen auf - $e$ ohne Umlaut schwankt bzw. von -er zu -e übergeht:

(1) -"er >-e: $\quad$ Bröter > Brote, Denkmäler > Denkmale, Stücker > Stücke.

Im letzteren Fall sind sowohl Maskulina als auch Feminina betroffen, deren Entwicklung verschieden ist. Die Maskulina geben den Umlaut auf und bilden den regulären Plural der starken Flexionsklasse auf -e, die Feminina geben nicht nur den Umlaut auf, sondern wechseln zu der für ihre Klasse regulären Pluralbildung mit -en:

\section{(2) -"e > -e: Gäuche > Gauche, Büge > Buge, Schlöte > Schlote, -"e>-en: $\quad$ Schlüchte > Schluchten, Süchte > Suchten, Flüchte > Fluchten}

Die Klasse der starken Feminina ist extrem klein, ${ }^{1}$ hochmarkiert und unproduktiv. Bei den starken Maskulina ist nicht unumstritten, ob der Umlautplural noch produktiv und ob er der markierte oder der unmarkierte Plural dieser Klasse ist, siehe Wurzel (1996), Elgersma/Houseman (1999). Nach den Zahlen in Köpcke (1993: 145, 149) ist der Umlautplural heute aber ebenfalls unproduktiv und markiert, ${ }^{2}$ siehe auch Eisenberg (1998), Thieroff (2002).

Die Unproduktivität des er-Plurals ist dagegen unumstritten (Wurzel 1984, Eisenberg 1998). Die meisten der folgenden in Paul (1917) und Mugdan (1977: 211ff). aufgeführten Beispiele

\footnotetext{
${ }^{1}$ Nach Pavlov (1995: 46) gibt es unter den Feminina 0,5 \% mit Umlautplural und 0,6 \% mit e-Plural (Types); bei letzteren handelt es sich um Ableitungen auf -nis.

2 Nach Köpckes Zahlen (1993: 144,148) hat die Produktivität des Umlautplurals im 20. Jahrhundert stark nachgelassen, s. die rückläufigen Zahlen in Tabelle 1. Während sich aus Mugdans Angaben (1977: 97) für das Ende des 19. Jahrhunderts (basierend auf dem Wörterbuch von Kaeding 1989) für den umgelauteten e-Plural noch ein Anteil von 32,8 \% und für den umgelauteten 0-Plural von 5,21\% ergibt (= Anteil an den Formen der starken Flexionsklasse), lassen sich aus Pavlovs Angaben (1995: 44f, basierend auf dem Wörterbuch von Wahrig 1975) nur noch die Werte 17,73\% für -" $e$ und 0,47 \% für -"0 errechnen. Es ist daher anzunehmen, dass sich z.B. für das Lehnwort Park, das derzeit die assimilierten Pluralformen Parke und Pärke (im Schweizerdeutschen) aufweist, im Standard die Form ohne Umlaut, nur in apokopierenden Dialekten die umgelautete durchsetzen wird.
} 
sind heute schon keine Zweifelsfälle mehr, sondern entweder obsolet oder laut Duden 2001 nur noch in bestimmten Dialekten gebräuchlich:

(3) er-Plurale

Antlitzer, Äser, Beeter, Beiler, Beiner, Better, Billetter, Blecher, Bröter, Buketter, Camisöler, Däuser, Departementer, Dinger, Einhörner, Elementer, Ender, Flösser, Gebeiner, Gebilder, Gemüser, Gerichter, Gesänger, Geschäfter, Gewerber, Gewichter, Gewölber, Gezelter, Handwerker, Hemder, Hirner, Hospitäler, Hundsfötter, Jöcher, Kabinetter, Kameler, Kleinöder, Klötzer, Kreuzer, Läuber, Lazarether, Lokäler, Mähler, Mäler, Menscher, Möser, Parlamenter, Rester, Röhrer, Rösser, Scheiter, Scheusäler, Seiler, Skandäler, Steiner, Stifter, Sträußer, Stükker, Testamenter, Törer, Viecher, Werker, Wichter, Zelter, Zeuger, Zieler.

(4) e+Umlautplurale

Ärme, Büge, Dächse, Flöre, Gäuche, Hälme, Hüfe, Hünde, Klöpse, Knäule, Lüchse, Moräste, Münde, Päcke, Päsche, Pastöre, Prähme, Pünsche, Röste, Rüfe, Schälke, Schlöte, Schlücke, Schmätze, Schüfte, Späte, Spünde, Täge, Törfe, Träume, Zwiebäcke

Angesichts des heute zu beobachtenden Abbaus stellt sich die Frage, wie es überhaupt zu diesen Formen und nun zur Variation zwischen markierter und unmarkierter Form kam. Sowohl der Umlautplural als auch der er-Plural sind in apokopierenden Dialekten weiter verbreitet als im Standard, und das zeigt auch eine der wesentlichen Motivationen für ihre Entstehung und ihre Ausbreitung. Die Motivation für beide markierten Pluralformen war eine Zweifache: 1. stellten sie eine Ersatzform für nicht hörbares $e$-Suffix in apokopierenden Dialekten und apokopierenden Zeitstufen des Deutschen dar (Fnhd), denn die Apokope führte zu nichtikonischen Nullformen: die *Land, die *Wald. Zu den älteren $e$-Pluralformen Lande, Walde, Hause, Hofe, Bade, Wolfe (erhalten in Ortsnamen wie Niederlande, Eberswalde, Burghausen, Baden, Bischofshofen, Wolfen (mit Dativendung - $n$ )), die aufgrund von Schwa-Apokope mit dem Singular zusammenfielen, wurde substitutiv ein er- oder ein Umlautplural gebildet: Länder, Wälder, Häuser, Bäder, Höfe, Wölfe.

Diese Strategie gilt heute noch für süddeutsche Dialekte, in denen finales -ə nicht artikuliert wird und die deshalb - er oder den Umlaut kompensatorisch für - $e$ einsetzen, sodass nebeneinander bestehen:

(5) Standard

Steine, Stücke, Reste, Brote

Hemden, Hefte, Dinge, Rosse,

Beine, Mahle, Ski

(6)
Alem., Bair., Frk., Hess., Pfälz., Schwäb.

Steiner, Stücker, Rester, Bröter,

Hemder, Hefter, Dinger, Rösser,

Beiner, Mähler, Skier

Süddt

Ärm, Täg.

Insbesondere bei Nomina, bei denen kein Umlaut möglich ist, bilden diese Dialekte mit genereller Schwa-Apokope häufig er-Pluralformen (s. (5) sowie Globüsser im Schweizerdeutschen, Lexiköner im Schwäbischen).

2. Die $e r$ - bzw. Umlautformen waren eindeutig, wogegen die $e$-Formen mit den Dativ Singular-Formen zusammenfielen: 
dem Lande - die Lande, aber dem Lande - die Länder

die Hunde - dem Hunde, aber die Wölfe - dem Wolfe

$\mathrm{Zu}$ bedenken sind die früher artikellos gebrauchten Dativ Sg-Formen, von denen sich nur die $e r$ - und die umgelauteten $e$-Pluralformen unterscheiden:

(9) zu Hause, zu Wasser und zu Lande, zu Grabe tragen, zu Leibe rücken, bei Lichte besehen, zu Buche schlagen - die Häuser, Länder, Gräber, Leiber, Lichter, Bücher.

(10) bei Hofe, zu Kopfe steigen, zu Grunde gehen - die Höfe, Köpfe, Gründe.

Das $e$-Suffix zur Bildung des Dativ-Singular wurde im Laufe des 20. Jahrhunderts obsolet (an dem ?Denkmale). Mit dem Aussterben von dativmarkierten Formen wie Hute, Lande, die nur noch in Redewendungen gebraucht werden (mit dem Hute in der Hand, zu Wasser und zu Lande), entfällt das Bedürfnis für eine vom Dativ distinkte Pluralbildung und auf die Unproduktivität des er-Plurals folgt die des Umlautplurals. ${ }^{3}$ Für beide Pluralformen besteht keine Motivation mehr. ${ }^{4}$

Insofern kann die heutige Unproduktivität und das Aussterben beider irregulären Pluralformen funktional erklärt werden: Mit dem Abbau des Dativ Singular-Markers -e (dem Brote > dem Brot) entfällt eine der beiden Motivationen für diese Arten der Pluralbildung, in nicht apokopierenden Varietäten entfällt auch die andere. Brote ist jetzt eindeutig Plural. Die Zweifelsfälle und die Normprobleme sind also eine Konsequenz des Abbaus markierter Klassen und damit eines natürlichen Sprachwandelprozesses.

Mit dem Abbau dieser irregulären Klassen entspricht die Entwicklung des Deutschen den Annahmen der Markiertheitstheorie: Unproduktive Klassen erhalten nicht nur keinen Nachschub mehr durch Neubildungen und folglich auch keinen Ersatz für aussterbende Wörter, sie verlieren auch Mitglieder an die großen, regulären und produktiven Klassen (cf. Wurzel 1984: 154 zu primärer Produktivität). Ein Flexionsklassenwechsel von kleinen irregulären zu großen regulären Klassen macht das System insgesamt einfacher. Das hat für das Deutsche zur Folge, dass von den heute noch vorhandenen sechs Pluralklassen nur noch vier produktiv sind:

\footnotetext{
${ }^{3}$ Cf. die Zahlen in Tabelle 1 im Anhang, die einen deutlichen Rückgang für die Pluralmarker -e+Umlaut und -er aufweisen. Innerhalb des 20. Jahrhunderts sinkt der Anteil des ersteren von 9,2 auf 3,8, der des letzteren von 2,3 auf $0,7 \%$ (= Anteil an allen Pluralformen, Types).

4 Der Abbau dieser Pluralklassen führt jedoch nicht immer zu Zweifelsfällen, die ja durch Anpassung der markierten Pluralform an die dominierende Flexionsklasse entstehen. In vielen Fällen sterben die Wörter mit erbzw. Umlautplural einfach aus, indem sie aus der Mode kommen: Bälger, Wämser, Gemächer, Mähler, Gäuche, Büge. Mit den Nomen, die nicht mehr gebraucht werden, weil sie durch modischere ersetzt werden (Bälger Kids), gehen dann auch die markierten Pluralformen unter.
} 
(11a) Pluralklassen des Deutschen insgesamt

\begin{tabular}{|c|c|c|c|c|c|}
\hline \multirow[t]{2}{*}{ FK } & \multicolumn{3}{|c|}{ Gen Sg } & \multirow[t]{2}{*}{ Nom Pl } & \multirow[t]{2}{*}{ Beispiel } \\
\hline & M & $/ \mathrm{N}$ & $/ \mathrm{F}$ & & \\
\hline 1. schwach & $-(e) n$ & $/ * 5$ & /- & $-(e) n$ & Uhr-en, Hure-n \\
\hline 2. stark & $-(e) s$ & & /- & $-(e)$ & Jahr-e, Fahrer- \\
\hline 3. speziell & $-s$ & & /- & $-S$ & Auto-s, Kid-s \\
\hline 4. gemischt & $-(e) s$ & & $/ *$ & $-e n$ & Staat-en, Dat-en \\
\hline 5. irregulär stark & $-(e) s$ & & / - & $-"(e)$ & Bärt-e, Gärten- \\
\hline 6. irregulär stark & $-(e) s$ & & /* & $-e r$ & Hühn-er, Kind-er \\
\hline
\end{tabular}

Daraus ergibt sich derzeit folgendes Bild für die Regularität der Pluralbildung im Deutschen: (11b) Reguläre und irreguläre Pluralklassen

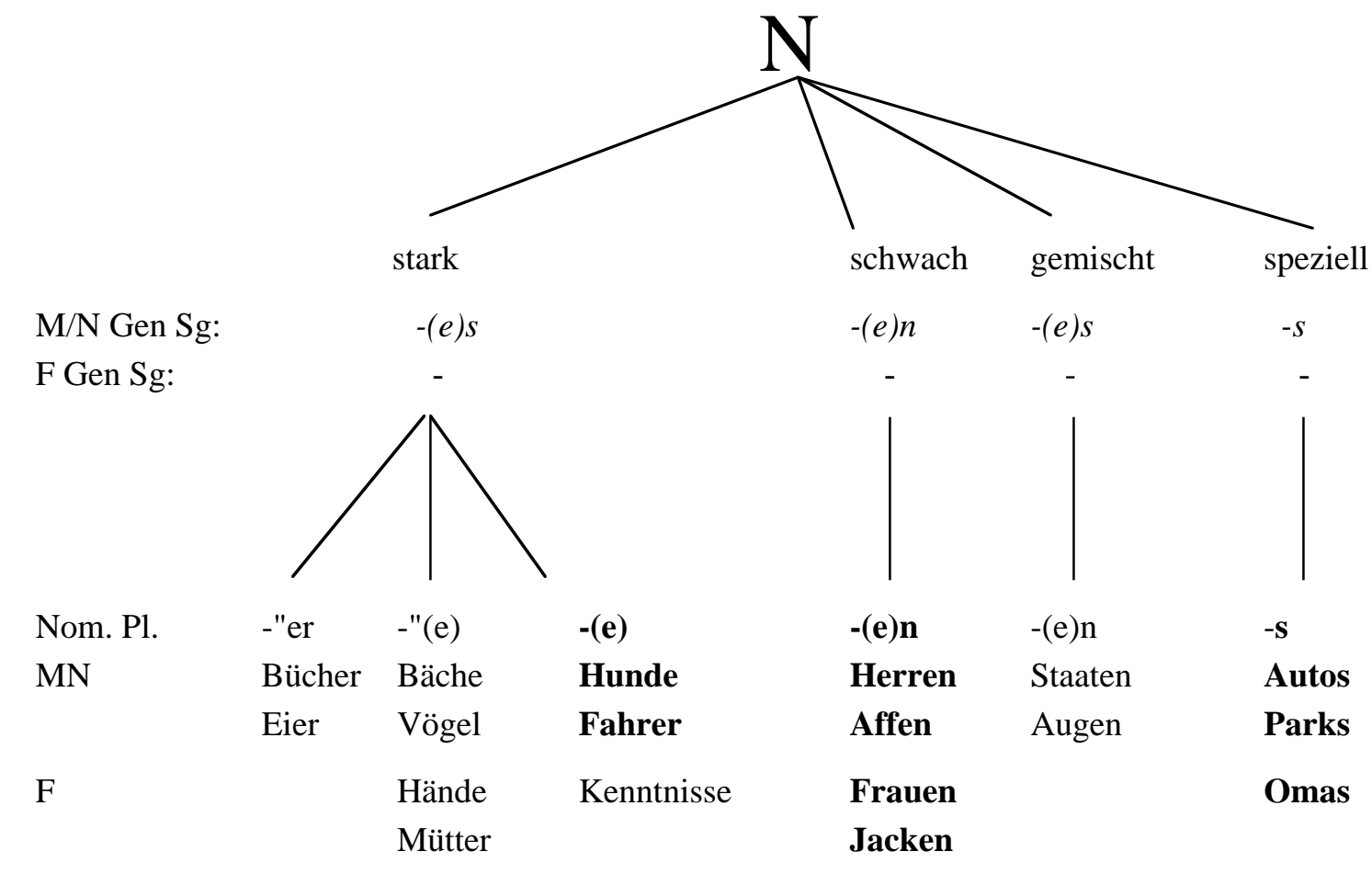

(11c) Produktive und unproduktive Klassen:

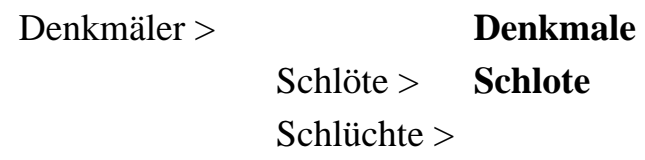

Bosse

Balkone

\section{Schluchten}

Boxen Globen Unis

$<$ Balkons

$<$ Mensas

Konten $\quad<$ Kontos

\footnotetext{
${ }^{5}$ Ein * bedeutet hier, dass das entsprechende Genus nicht vertreten ist.
} 
Insbesondere für Dialektsprecher besteht naturgemäß bisweilen Unsicherheit darüber, welche der beiden Formen die korrekte ist, diejenige, die in der dialektal gefärbten Umgangssprache, oder diejenige, die in standardsprachlichen überregionalen Texten, etwa den Medien, gebraucht wird. Im allgemeinen ist aber schon durch die Aussprache klar, was Dialekt, was Standard ist.

Das zeitweilige Schwanken einiger Nomen, die noch Formen der irregulären Klasse aufweisen, aber schon über Formen der regulären Pluralbildung verfügen, ist also eine unvermeidliche Begleiterscheinung des Abbaus von Markiertheit, und dies ein natürlicher Sprachwandelprozess.

Im Falle dieser beiden unproduktiv gewordenen Pluralklassen lässt sich mit einiger Sicherheit vorhersagen, welche der Formen überleben wird. Aufgrund von Analogiewirkung der großen Klassen und der geringeren Transparenz der umgelauteten Formen dürfte das die reguläre Form auf - $e$ bzw. - en sein. Das bedeutet nun aber nicht, dass alle "alten" er-bzw. "e-Formen aussterben werden. Der Umlaut verleiht ihnen größere Salienz, was eine besonders distinkte Pluralbildung ermöglicht, und das prädisponiert sie dazu, für Nomina im Hochfrequenzbereich zu überleben. Dass sie in diesem Bereich schon heute besonders stark vertreten sind, zeigt die Berechnung des Token:Type-Verhältnisses der Pluralklassen aus den Werten für 1975 von Tabelle 1 und 2 im Anhang. Während die Tokenfrequenz der irregulären Pluralformen deren Typefrequenz um ein Mehrfaches übersteigt, liegen die Werte der nativen $(e)$ - und (e)n-Plurale nahe 1, sind also ausgeglichen:

Token: Type-Quotient der Pluralklassen:

\begin{tabular}{llllll}
$\stackrel{-s}{-}$ & $-(e) n$ & $-(e)$ & irr. $-(e) n$ & $-"(e)$ & $-e r$ \\
\hline 0,17 & 0,82 & 0,91 & 2,29 & 3,94 & 10,14
\end{tabular}

Die hohen Werte zeigen hohe und extrem hohe Gebrauchsfrequenz für den Umlaut- und den er-Plural an. Das ist für markierte, durch den Umlaut schwach suppletive Formen normal, referieren solche Nomen doch in der Regel auf Entitäten des Nahbereichs des Menschen, s. Wurzel (1990). Die frequentesten dieser Wörter können dadurch als Ausnahmen überleben.

Hinzu kommt, dass in süddeutschen Varietäten und/oder bei Bezeichnungen für männliche Personen die Präferenz für den nicht umgelauteten (e)-Plural schwächer ist, was den Umlautplural stützt. Dadurch kann es auch bei Fremdwörtern außer zum Schwanken zwischen $s$ - und Schwa-Plural (s.u.) noch zu Schwankungen zwischen Umlautplural und reinem (e)-Plural kommen, z.B.

(13a) Generale - Generäle (nach Duden 2001 gleichwertig, nach meiner Beobachtung die e-Form eher bei älteren Sprechern' ${ }^{6}$,

(13b) Parke - Pärke (nach Duden 2001 gleichwertig und beide gegenüber Parks selten, die "e-Form in der Schweiz)

\footnotetext{
${ }^{6}$ Hörbeleg im Nov. 2002 von einem Teilnehmer des 2. Weltkrieges, Fernsehdokumentation.
} 


\subsection{Reparaturstrategien}

Im Gegensatz zur Unproduktivität des Umlautplurals steht die Tatsache, dass in gesprochenen Varietäten besonders süddeutscher Sprecher der Umlaut auch heute noch als kompensatorisches Mittel eingesetzt wird, so dass wir bei einigen Substantiven mit finaler Schwa-Silbe, die regulär den Nullplural bilden, Variation zwischen einer overten, wenn auch nur umgelauteten, und einer Null-Pluralform finden. Wegen der relativ starken Beschränkung des Deutschen auf trochäische Pluralformen und wegen der strikten Vermeidung der Aufeinanderfolge zweier Schwa-Silben können starke maskuline und neutrale Nomen mit finaler Schwa-Silbe keinen overten e-Plural bilden, der für ihre Klasse das reguläre Pluralsuffix darstellt (*Engele, *Fenstere). Im Gegensatz zu den schwachen Maskulina und den Feminina, die in diesem Falle immer noch einen nichtsilbischen $n$-Plural bilden können (Löwe-n, Ampel-n), ist die Pluralbildung bei Maskulina und Neutra blockiert und es kommt zum Null-Plural: Engel-, Fenster-.

Für diesen Verstoß gegen das Ikonismusgebot treten in der Umgangssprache mehrere Reparaturstrategien auf. Bei hinteren Vokalen kann es zur Umlautung des Stammvokals kommen, was besonders häufig beim unmarkiertesten unter den hinteren Vokalen, nämlich [a], eintritt, in anderen Fällen kommt es zur Addition von -s (auch zusätzlich zu -en) oder zur Addition von $-n$, so dass wir folgende Varianten finden:

(14) Standard Süddt

Wagen, Kragen, Kasten, Bogen, Magen Wägen, Krägen, Kästen, Bögen, Mägen,

Faden, Laden, Hammel, Hammer, Stadel $\quad$ Fäden, Läden, Hämmel, Hämmer, Städel

(Schweiz)

(15) Standard Norddt

Mädel, Kumpel, Onkel, Bengel,

Tunnel, Jungen

Mädels, Kumpels, Onkels, Bengels,

Tunnels, Jungens

(16) Standard

Österreich., Süddt

Onkel, Spiegel, Spargel, Mädel,

Stadel

Onkeln, Spiegeln, Spargeln, Madln,

Stadeln

Der $s$-Plural der norddeutschen Varianten wird in dieser Funktion schon seit dem 17. Jahrhundert, d.h. seit seiner Verfügbarkeit im Deutschen, für Maskulina und Neutra verwendet, die auf eine Schwa-Silbe auslauten (s. Paul 1917) und aus prosodischem Grund keinen silbischen Plural ausbilden können. Wie der er-Plural und der Umlautplural bei den Einsilbern in (5) und (6) haben der Umlaut, der $s$ - oder der $n$-Plural hier eine Ersatzfunktion, stellen eine Reparaturstrategie für den 0-Plural dar. Im Gegensatz zu er- und Umlaut-Plural konnte sich allerdings bisher keine dieser $s$ - oder $n$-Pluralformen im Standard durchsetzen. Das Schwanken zwischen zwei Formen beruht hier also nicht auf dem Abbau der einen Klasse zugunsten einer anderen, sondern genau umgekehrt auf der Tatsache, dass sich die overten Formen nicht definitiv etablieren konnten und noch immer als "umgangssprachlich" gelten, s. Duden 2001 für Onkel. Da keine dieser Formen in der Normsprache die unmarkierten Nullformen verdrängen konnte, besteht hier teilweise schon seit zwei- bis dreihundert Jahren Variation. Diese Fälle dürften neben denen in 2.3 die langlebigsten Zweifelsfälle der deutschen Sprache sein. 
Einige dieser Formen sind schon im 18. Jahrhundert bei Goethe und Schiller belegt, siehe Paul 1917:

(17) s-Plurale:

Briefchens, Briefleins, Bubens, Damens, Engelchens, Engels, Fräuleins, Jungens, Kinderchens, Mädchens, Mädels, Ministers, Möbels, Mütterchens, Onkels, Paketchens, Pfarrers, Schlingels, Thälerchens, Titels

(18) n-Plurale

Ziegeln, Spiegeln, Stadeln, Fusseln, Bröseln, s. auch 2.3

Bei der Verwendung des n-Plurals für starke Maskulina und Neutra kann es sich allerdings immer auch um Überreste einer ehedem schwachen Flexion dieser Nomina handeln, s. 2.3.

\subsection{Flexionsklassenwechsel aufgrund von Resemantisierung der schwachen Maskulina}

Die Klasse der schwachen Maskulina wird derzeit (siehe Köpcke 1995) resemantisiert, mit der Folge, dass Nomina mit dem Merkmal [+ belebt] in diese Klasse aufgenommen (des Autors > des Autoren), Nomina ohne dieses Merkmal dagegen aus ihr ausgeschlossen werden. Im Fall von Nomina wie Funke, Gedanke... (Wurzel 1985) geschieht dies durch Addition des obliquen $-n$ an den Nominativ, ohne dass sich die Pluralform selbst ändert. Sie wird nur von einem $n$-Plural zu einem Null-Plural reanalysiert, so dass hier keine Zweifelsfälle hinsichtlich des Plurals, wohl aber solche hinsichtlich des Nominativ Singulars bestehen:

\begin{tabular}{lll}
\multicolumn{2}{c}{ Sg } & Plural \\
\hline alt: & & \\
$\mathrm{N}$ & Funke & Funke-n \\
$\mathrm{G}$ & Funke-ns & Funke-n \\
$\mathrm{DA}$ & Funke- $n$ & Funke-n \\
neu: & & \\
$\mathrm{N}$ & Funken & Funken- \\
$\mathrm{G}$ & Funken-s & Funken- \\
$\mathrm{DA}$ & Funken & Funken-
\end{tabular}

Ebenso schwankt der Nom Singular bei Buchstabe, Gedanke, Glaube, Name, Wille zwischen einer Form mit und einer ohne finales - $n$, s. Köpcke (1995: 173).

Bei denjenigen Nomen, die bereits den Genitiv auf $-s$ bilden (des Ziegels, des Stiefels) und somit zur gemischten Flexionsklasse gehören (s. Paul 1917), wird teilweise die nun nicht mehr durch das NP des implikativen Paradigmenaufbaus IPA (s.u.) motivierte Pluralform auf - $n$ abgebaut und durch eine solche mit dem Null-Plural ersetzt (die Ziegel, die Stiefel). Hier kommt es also zum Schwanken zwischen einer Form der starken und einer der schwachen Flexion, rein formal ebenfalls wie in (16), (18) oben zwischen Null und - $n$. Betroffen sind vor allem Nomen mit Auslaut auf -el: 
(20) Stiefel - Stiefeln, ebenso Fussel, Model, Ziegel, Stadel, ${ }^{7}$ s. Mugdan (1977: 211).

Da hier keine Reanalyse des Plurals erfolgt wie bei Funke- $n>$ Funken-, sondern das - $n$ getilgt wird, hat dieser Übertritt in die starke Flexionsklasse keine Auswirkung auf den Nominativ oder Genitiv Singular, der bereits angepasst ist.

Auch diese Zweifelsfälle bestehen schon seit Jahrhunderten, s. Paul (1917: 57ff). Sie sind auch deshalb relativ langlebig, weil der semantisch motivierte Übergang in die starke Flexionsklasse zum Nullplural führt, der aber natürlich gegen das NP des konstruktionellen Ikonismus verstößt und als schlecht empfunden wird, wie die in 2.2 dokumentierten Reparaturstrategien zeigen.

Bei belebten Nomen siegt in diesen Fällen i.a. der $n$-Plural, teilweise gehen sie definitiv zur schwachen Flexion über und bilden auch den Genitiv auf - $n$ : Bauern, Bayern, Pommern, aber Vettern mit $s$-Genitiv.

Bei unbelebten Nomen siegt in diesen Fällen heute meist trotz seiner Nicht-Ikonizität der Null-Plural. Dieser ist für starke Nomina mit finaler Schwasilbe also nicht unproduktiv. Er wird auch für die große und prinzipiell offene Zahl von Nomina agentis auf -er (der Fahrer, Besucher, Sprecher...) und für Fremdwörter mit diesem Suffix (Gangster, Computer...) produktiv eingesetzt. Mit der Verwendung des Null-Plurals in all diesen Fällen ist ein anderes Markiertheitsprinzip erfüllt, das des implikativen Paradigmenaufbaus IPA (Wurzel 1984: 116ff) - der Genitiv Singular ist aus dem Nominativ Plural implikativ ableitbar und umgekehrt, die Formen Paradigmas implizieren sich gegenseitig:

$\begin{array}{lll}\text { Nom } \mathrm{Pl} & \leftrightarrow & \text { Gen } \mathrm{Sg}: \\ \text { die Hunde } & \leftrightarrow & \text { des Hundes, } \\ \text { die Affen } & \leftrightarrow & \text { des Affen. }\end{array}$

Auch diese langlebigen Zweifelsfälle sind also nur Effekte eines natürlichen Sprachwandelprozesses. Da sowohl die starke als auch die schwache Flexionsklasse produktiv sind, sind prinzipiell Übertritte in beide Richtungen zu erwarten. Derzeit scheint die Tendenz zu überwiegen, unbelebte Nomen stark, belebte Nomen (zumindest solche mit bestimmten phonologischen Merkmalen, s. hierzu den Beitrag von Thieroff in diesem Band) schwach zu flektieren. Es kann daher angenommen werden, dass sich bei unbelebten Nomen der nicht-ikonische Null-Plural durchsetzt, zumindest im Standarddeutschen.

\section{$3 \quad$ Fremdwörter}

Die größte Zahl von Zweifelsfällen besteht erwartungsgemäß bei aus fremden Sprachen entlehnten Wörtern, Fremdwörtern also. Wenn diese "eingedeutscht", d.h. ins Deutsche integriert werden, ändern sie nicht nur ihre Aussprache und Orthographie, sondern auch ihr Flexionsverhalten. Sofern es sich um flektierbare Wörter handelt, entwickeln sie assimilierte Flexionsendungen und -formen, im Fall der Substantive Kasus- und Pluralformen. Dabei können un-

\footnotetext{
${ }^{7}$ In zwei dieser Fälle kommt es zu schwankenden Pluralformen außerdem durch schwankendes Genus, so bei Fussel und Model [moldel], die als Maskulina 0-Plural, als Feminina aber natürlich $n$-Plural haben und damit die Regularität dieser Pluralsuffixe belegen.
} 
ter Umständen drei verschiedene Formen auftreten, die drei unterschiedlichen Stufen im Assimilationsprozess entsprechen.

Fremdwörter können mit ihrem ursprünglichen, also dem Suffix der Gebersprache, pluralisiert werden, das bei Klassizismen und Italianismen einen Vollvokal aufweist:

(22a) Visa, Antibiotika, Kommata, Pizze, Celli, Spaghetti,

bei Anglizismen und Gallizismen dagegen ein unsilbisches $-s$ ist:

(22b) Songs, Bars, Lifts, Tests, Balkons,

oder sie werden, nach erfolgter Assimilation, mit einem Schwa-Suffx pluralisiert:

(22c) Visen, Pizzen, Lifte, Teste, Balkone.

Interessanterweise tritt das $s$-Suffix auch bei einigen Klassizismen und Italianismen auf, bei denen es also nicht aus der Gebersprache mitentlehnt sein kann:

(22d) Kommas, Themas, Visas, Kontos, Pizzas.

Wenn hier neben Formen mit einem fremden Pluralsuffix solche mit $s$-Plural und solche mit bereits assimiliertem nativem Pluralsuffix bestehen, so haben wir drei Formen, nämlich fremde, unassimilierte und assimilierte Pluralformen, nebeneinander. Dabei kann die Stufe mit $s$ Plural auch als teilassimiliert gelten, anders gesagt, der $s$-Plural stellt dann eine Zwischenstufe im Assimilationsprozess, einen Übergangsplural dar.

(23) Schwanken zwischen 3 Formen:

$\begin{array}{lll}\text { fremder Plural } & \text { s-Plural } & \text { assimilierter Plural } \\ \text { Schemata } & \text { Schemas } & \text { Schemen } \\ \text { Themata } & \text { Themas } & \text { Themen } \\ \text { Pizze } & \text { Pizzas } & \text { Pizzen } \\ \text { Mensae } & \text { Mensas } & \text { Mensen } \\ \text { Visa } & \text { Visas } & \text { Visen } \\ \text { Lexika } & \text { Lexikas } & \text { Lexiken } \\ \text { Conti } & \text { Kontos } & \text { Konten }\end{array}$

(24) Schwanken zwischen 2 Formen:

\begin{tabular}{|c|c|c|c|}
\hline fremder Plural & & s-Plural & assimilierter Plural \\
\hline- & a) & Lifts & Lifte \\
\hline - & & Fracks & Fräcke \\
\hline- & & Scheichs & Scheiche \\
\hline & & Balkons & Balkone \\
\hline & & Tests & Teste \\
\hline & & Parks & Parke \\
\hline (généraux) & & Generals & Generäle \\
\hline
\end{tabular}




\begin{tabular}{|c|c|c|c|}
\hline - & b) & Saunas & Saunen \\
\hline - & & Dramas & Dramen \\
\hline . & & Datschas & Datschen \\
\hline & & Pesetas & Peseten \\
\hline - & & Astas & Asten \\
\hline & & Kontos & Konten \\
\hline - & & Albums & Alben \\
\hline - & & Taxis & Taxen \\
\hline & & Sozis & Sozen \\
\hline - & & Tussis & Tussen \\
\hline Cakes $^{8}$ & c) & Keks & Kekse \\
\hline- & & Straps & Strapse \\
\hline - & & Pumps & Pumpse \\
\hline - & & Shrimps & Shrimpse \\
\hline & & Chips & Chipse \\
\hline
\end{tabular}

Die $s$-Zwischenstufe ist im Falle der Anglizismen und Gallizismen im allgemeinen identisch mit dem fremden, englischen oder französischen Plural, weshalb dieser als aus der Gebersprache mitentlehnt erscheint. Das ist jedoch nicht immer der Fall, s. Generals. Der Übergang von scheinbar mitentlehnten $s$-Formen zu $e$-/en-Formen könnte nun einfach dadurch erklärt werden, dass zunächst die fremde, mitentlehnte Pluralform gebraucht wird und diese dann im Zuge der Assimilation durch die der nativen Pluralbildung entsprechende Form ersetzt wird, ebenso wie Aussprache und Orthographie angepasst werden. Damit wäre die Entwicklung nur durch Anpassungsdruck der großen Klassen bedingt. Sie könnte unabhängig von der Struktur der Formen so verlaufen, dass stets die dominante native Klasse die kleinere "fremde" Klasse aufsaugt, müsste also im Deutschen von $-s>-(e) /-(e) n$, im Englischen aber von $-(e) /-(e) n>-s$ verlaufen, wie dt Leitmotive, e. Leitmotifs zeigen. Durch reine Anpassung können jedoch die $s$-Formen in (23) und (24b) und vereinzelt in (24a) (Generals) nicht erklärt werden, denn diese Formen für Nomina, deren Plural im Original ein fremdes Suffix (- $i,-e,-t a,-a e)$ aufwies, sind unzweifelhaft erst innerhalb des deutschen Sprachgebiets ausgebildet worden.

Insbesondere die dreistufige Entwicklung wirft die Frage auf, warum im Deutschen z.B. für das Fremdwort Pizza, für das nach Wahrig 1968 zunächst der fremde, italienische Plural Pizze (mit finalem [e]) verwendet und dann "eindeutschend" Pizzas gebraucht wurde, nicht direkt die phonetisch näherliegende Form Pizzen gebildet wurde, warum das Deutsche also vor der Bildung der assimilierten Form die Übergangsform Pizzas ausbildet - und warum es diese wieder aufgibt. Warum verläuft die Assimilation von Pizze zu Pizzen nicht direkt von -e zu $e n$, was phonologisch nahe liegt, sondern von -e über -as zu -en, also -e > -as > -en, warum dieser Umweg über die $s$-Form?

Im Falle der zweistufigen Entwicklung stellt sich entsprechend die Frage, warum das Deutsche nicht seine produktiven Pluralsuffixe sofort einsetzt und gleich die Formen Balkone, Lifte, Generale / Generäle, Rivalen ausbildete. Zweifelsfälle wären zwar auch dann unvermeidlich, aber wir hätten dann nur Normprobleme zwischen fremdem und nativem Suffix.

\footnotetext{
${ }^{8}$ Ein Plakat zur Weltausstellung 1900 wirbt für Leibniz-Cakes der Hannoverschen Cakes-Fabrik H.Bahlsen.
} 
Die Tatsache, dass für Klassizismen und Italianismen "eindeutschend" ein $s$-Plural gebildet wird, wirft die Frage auf, ob dieser bei Anglizismen und Gallizismen wirklich mitentlehnt oder nicht auch erst von deutschen Sprechern ausgebildet wird. Für diese Annahme spricht,

1. dass das $s$-Suffix im gesprochenen Französisch nicht artikuliert wird,

2. dass $-s$ im Deutschen auch bei Ausnahmen auftritt, die in der Gebersprache einen irregulären Plural aufweisen (frz généraux > deutsch Generals),

3. dass es nicht automatisch bei allen Anglizismen und Gallizismen auftritt, auch wenn die Originale einen $s$-Plural haben (engl. gangsters, faxes $>$ dt. Gangster/*Gangsters, Faxe/ *Faxes, französ. ingénieurs > deutsch Ingenieure/*Ingenieurs). Seine Anwendung durch deutsche Sprecher muss also erklärt werden.

Der $s$-Plural kann vor allem deshalb nicht wie die italienischen, griechischen, lateinischen Suffixe als "fremder" Plural gelten, weil er im Gegensatz zu diesen

4. auch bei Fremdwörtern auftritt, die ihn in der Gebersprache nicht aufweisen.

Vielmehr ist davon auszugehen, dass der $s$-Plural unabhängig von den Verhältnissen in der Gebersprache von deutschen Sprechern ausgebildet oder zumindest bestätigt wird. Daraus ist zu schließen, dass er bestimmte Beschränkungen erfüllt, die im Deutschen gelten, und dass er da, wo er nicht oder nicht mehr verwendet wird, anderen im Deutschen gültigen Beschränkungen unterliegt, die höher gerankt sind als die, die die $s$-Pluralformen optimal erfüllen.

Der Assimilationsprozess umfasst also zwei Schritte, die Bildung bzw. Verwendung des $s$ Plurals und seinen Abbau. (Zur Erklärung sowohl der Ausbildung als auch des Abbaus des sPlurals s. genauer Wegener 2002). Da beide Schritte nicht abrupt erfolgen, kommt es während der Entstehungs- ebenso wie während der Abbauphase zu Schwankungen und damit zu Normproblemen.

\subsection{Schwanken zwischen fremdem Plural und teilassimiliertem Plural auf -s}

Die Formen mit fremdem Suffix werden im allgemeinen nur in fachsprachlichen und Prestigevarietäten gebraucht, sie sind relativ selten und markiert, daneben existieren umgangssprachlich häufig Formen mit $s$-Plural:

(25a) Celli, Motti, Espressi, Themata, Kommata, Schemata, Lexika, Pizze.

(25b) Cellos, Mottos, Espressos, Themas, Kommas, Schemas, Lexikons, Pizzas.

Die Erklärung für die Verwendung der fremden Pluralformen dürfte soziolinguistischer Natur sein: In bestimmten Fachkreisen zeichnet es den Fachmann aus, wenn er sich dieser Formen bedient, weil er damit sein Wissen dokumentiert und seine Zugehörigkeit zu einer bestimmten Gruppe von Fachleuten demonstriert. Die fremden Pluralformen beweisen Bildung und besitzen daher Prestige.

Geht das Fremdwort mit dem fremden Pluralsuffix in die Umgangssprache ein, so kann es zur Reanalyse und Ausbildung eines sekundären Plurals, also einer Doppelpluralform kommen, s.u. zu Fällen wie Spagettis und Praktikas. 


\subsection{Schwanken zwischen s-Plural und Schwa-Plural}

Die Formen mit $s$-Suffix gehen denjenigen mit Schwa-Suffix voraus. Sie stellen die erste Stufe des Assimilationsprozesses dar, denn diese verläuft stets von einer eventuell vorhandenen Stufe mit fremdem Suffix zu einer Stufe mit $s$-Suffix und von dieser zur definitiven, endgültigen Stufe mit Schwa-Suffix. Während es keiner besonderen Erklärung dafür bedarf, dass assimilierte Formen native Suffixe verwenden, bedarf die Ausbildung bzw. Verwendung des $s$ Plurals einer Erklärung. Diese hat die innere Struktur des Fremdworts zu berücksichtigen, d.h. die Frage, ob es sich bei der Entlehnung um ein morphologisch gegliedertes oder ein morphologisch einfaches Substantiv handelt. Ersteres liegt im allgemeinen bei Klassizismen und Italianismen, letzteres bei Anglizismen und Gallizismen vor.

\subsubsection{Klassizismen und Italianismen}

Die Ausbildung des $s$-Plurals kann hier funktional erklärt werden, wenn man sich die Struktur des Singularstamms der betroffenen Fremdwörter klar macht:

(26) Conto, Espresso, Thema, Pizza, Album, Globus, Ritus, Lexikon, Mythos, Praxis

Die Entlehnungen aus dem Italienischen und Griechischen enden teilweise auf Vollvokal und sind in jedem Fall morphologisch komplex, d.h. sie bestehen aus einer Wurzel und einem Stammbildungssuffix (- $a,-o,-u m$, -on, os, $-u s,-i s)$. Beides erschwert die Assimilation. Das soll am Beispiel von Pizza demonstriert werden.

Als Femininum verlangt Pizza den en-Plural. Die einfache Addition des en-Suffixes würde hier aber zu einem Hiat und zu einem dreisilbigen Wort führen, ist deshalb ungrammatisch: ${ }^{9}$ *'Pizzaen. Als assimilierte Form mit Schwa-Plural ist nur Pizzen möglich. Diese Form aber verlangt, dass der Endvokal des Singularstamms gekappt wird. Tilgung von Segmenten des Stamms verstümmelt aber den Stamm, stellt eine Verletzung des Transparenzprinzip dar. Als Notlösung wird Pizzas gebildet, obwohl diese Form mit gespanntem [a] gegen eine phonologische, im Deutschen nativen Wortschatz allgemein gültige Beschränkung verstößt, derzufolge Vokale in unbetonten geschlossenen Silben ungespannt sind, cf. Kokos [kokəs], Humus [humus], Ananas [ananas], Königin [kønigin].

Pizzas verändert den Singular Pizza nicht, es bewahrt sogar die Gespanntheit des Vokals, obwohl dieser im Plural nicht mehr in offener, sondern in geschlossener Silbe steht. Dass die Entspannung des Vokals hier nicht auftritt, Pizzas [a] nicht wie Ananas mit [a] artikuliert wird, das dann bis zum Schwa reduziert werden könnte, zeigt, dass die Assimilation nicht als allmähliche phonologische Anpassung, sondern in einem Sprung erfolgt. Dieser Sprung stellt einen abrupten Übergang und einen Wechsel des Flexionstyps von Grundformflexion zu Stammflexion dar. Bevor dieser Schritt erfolgt, ist Pizzas als Übergangsplural optimal, denn der $s$-Plural ermöglicht eine strukturbewahrende, transparente Pluralform.

Die assimilierte Form Pizzen mit der Tilgung des finalen Vollvokals setzt die Klassifizierung dieses Vokals als Suffix voraus: Vill-a-en, Pizz-a-en. Vokaltilgung kann daher erst erfolgen,

\footnotetext{
${ }^{9}$ Hiat ist nur in betonten Silben möglich, cf. I'deen vs *'Unien
} 
wenn der finale Vokal als Stammbildungssuffix erkannt ist, sich bei den Sprechern der entlehnenden Sprache ein Morphembewusstsein herausgebildet hat für die Tatsache, dass mit Pizza ein morphologisch komplexes, intern strukturiertes Nomen vorliegt. Ein solches Morphembewusstsein kann zu Anfang der Verwendung eines Fremdworts bei den Sprechern der entlehnenden Sprache nicht vorhanden sein, es muss sich erst entwickeln. Solange die Sprecher Villa, Pizza als monomorphematisch betrachteten, mussten sie also die Formen Villas, Pizzas ausbilden. Denn solange der Endvokal für die Sprecher des Deutschen als Teil des Stammes gilt, kann das Pluralsuffix nur an den Stamm $\{\text { Pizza }\}_{\mu}$ hinzugefügt werden, also ist wegen des Vollvokals nur $-s$ möglich. Anders gesagt: Solange Pizza als einmorphemig gilt, kann kein Suffix getilgt werden, da für den Sprecher kein Suffix vorliegt. Die Bildung einer $s$ Pluralform ist die Konsequenz der Unteranalyse eines eigentlich morphologisch komplexen Wortes. Die Beibehaltung des gespannten Vokals in der nun geschlossenen Silbe ist eine Folge des Bemühens um Morphemkonstanz.

Im Sprecherbewusstsein durchläuft das Nomen sodann eine Entwicklung von einem monomorphematischen zu einem intern gegliederten Nomen:

$$
\{\text { Pizza }\}_{\mathrm{St}}>\{\text { Pizz }\}_{\mathrm{Wz}}\{\mathrm{a}\}_{\mathrm{Sf}}{ }^{10}
$$

Erst wenn den Sprechern die komplexe morphologische Struktur des Nomens bewusst ist, kann dieses in Stamm und Suffix zerlegt und das Pluralsuffix an den Stamm $\{\text { Pizz- }\}_{\mu}$ treten, folglich kann -en appliziert, Pizzen gebildet werden. Bewusstsein vom Suffixstatus der Endung kann sich durch den Vergleich mit anderen stammflektierenden Formen (Firma Firmen) entwickeln oder aus Kenntnis der Gebersprache geschöpft werden (s. Harnisch 1994). Bei intern gegliederten, vom Sprecher aber nicht als komplex erkannten Fremdwörtern ist $-s$ das einzig mögliche Pluralsuffix, wenn die Nomen auf Vollvokal auslauten. Insofern ist $-s$ hier sowohl ein Notplural als auch ein Transparenzplural, der, indem er das Kappen der Endung umgeht, einen höheren Grad an Strukturbewahrung erlaubt als der endgültige en-Plural.

Bei Neutra und Maskulina wie Album, Ritus wäre zwar ein $e$-Suffix phonologisch möglich, um den Preis einer dreisilbigen Pluralform (*Albume, *Ritusse), jedoch gehen diese Nomen definitiv zur Stammflexion über und bilden die Pluralformen Alben, Riten aus. Wenn der $s$ Plural bei Latinismen auf -um auftritt, vermeidet er entweder die Entstehung einer nicht trochäischen Pluralform oder Stammflexion mit Kappen des Suffixes:

(28b) Albums vs *Albume vs Albumen.

Bei morphologisch gegliederten Wörtern, Entlehnungen also aus den klassischen Sprachen und dem Italienischen, oder solchen, die deutsche Sprecher so klassifizieren, ${ }^{11}$ die letztendlich zur Stammflexion im Deutschen übergehen, verhindert das $s$-Suffix also entweder die Bildung

\footnotetext{
${ }^{10} \mathrm{St}=$ Stamm, Wz $=$ Wurzel, $\mathrm{Sf}=$ Stammsuffix.

11 Stammflexion im Deutschen ist nicht davon abhängig, ob das Fremdwort in der Quellsprache morphologisch komplex ist oder nicht. Für das aus dem Finnischen entlehnte Sauna ist entscheidend, dass es von deutschen Sprechern aufgrund seines "lateinischen" Auslauts als komplex interpretiert wird. Stammflexion tritt tatsächlich eher ein, wenn das Wort aus einer klassischen, slawischen oder romanischen Sprache entlehnt wurde, als bei Exotismen wie Geisha, s. Harnisch (1994).
} 
eines Hiats, die Entstehung einer dreisilbigen Pluralform oder die Tilgung der Endung des Singularstamms. Die mit $-s$ auftretende Grundformflexion hält die Singularform des Fremdworts vorübergehend intakt. Hier finden wir daher auch Schwanken zwischen Grundformflexion und Stammflexion: ${ }^{12}$

(28b) Pizza-s - Pizz-en, Album-s - Alb-en

Nur bei Maskulina auf -us kommt es auch im Fall der Grundformflexion zu einer finalen Schwa-Silbe, wogegen die Stammflexion das Kappen der Endsilbe des Singularstamms, des Stammbildungssuffixes - us impliziert:

(29) Zirkusse, Krokusse vs Rhythmen, Riten, Mythen und als Varianten Globusse - Globen.

Einen Sonderfall stellt Atlas mit den Pluralformen Atlanten und Atlasse dar. Ersteres ist eigentlich die Pluralform von Atlant, weshalb es nur konsequent ist, wenn sich die reguläre Form Atlasse durchsetzt.

Im Extremfall kann der Zweifel an der korrekten Pluralform sogar zu einer Blockierung der Pluralbildung führen: Im Augenblick ist nach Duden (2001) nicht klar, wie für das Wort Campus ein Plural gebildet werden soll. Duden gibt hier nicht gerechtfertigten 0-Plural an, meine Studenten schlagen Campusse vor. Ähnliches gilt für Ananas, dessen Plural nach Duden (2001) derzeit zwischen 0- und $e$-Plural schwankt, die beide nicht der Flexionsklasse für ein Femininum entsprechen: Ananas - Ananasse.

(30) Campus- - Campusse, Ananas- - Ananasse

Problematisch im Hinblick auf das IPA sind alle gemischt flektierenden Nomen. Besonders problematisch ist jedoch die Tatsache, dass diese Klasse irregulärer Plurale produktiv ist. Zuwachs bekommt die gemischte Flexionsklasse durch die Maskulina und Neutra unter den Fremdwörtern aus den klassischen Sprachen und dem Italienischen, die morphologisch komplex sind und via Stammflexion integriert werden (31a):

(31a) Rit<us>en, 'Anglizism<us>en, Myth<os>en, Stadi<on>en, Mu'se<um>en, Kont $<o>e n$.

Das erlaubt zwar die Ausbildung trochäischer Pluralformen gegenüber den daktylischen der Grundformflexion (Zirkusse). Jedoch bilden die Maskulina und Neutra, die im Singular stark flektieren, wider Erwarten keinen $e^{-}$, sondern einen $e n$-Plural (31b), den Stammflexionsplural des Deutschen:

(31b) des Kontos - die Konten, des Globus - die Globen, nicht *Konte, *Globe.

Mit dem einheitlichen Suffix erfüllen diese Nomen zwar das Natürlichkeitsprinzip der Uniformität, durch Verletzung des IPA stellen sie aber ein Subsystem innerhalb der deutschen Nominalflexion dar. ${ }^{13}$ Dessen Irregularität wird geduldet, weil diese Nomen über relativ zuverlässige Genusmarker verfügen, welche zugleich die Flexionsklasse anzeigen:

\footnotetext{
12 Nach Harnisch (1994: 106) erfolgt Stammflexion bei $66 \mathrm{M}$ auf - $u$ s zu fast 50\%, bei $78 \mathrm{~N}$ auf -um zu fast 100 $\%$, bei $26 \mathrm{~N}$ auf $-a$ zu mehr als $50 \%$, bei $69 \mathrm{~F}$ auf $-a$ zu mehr als $67 \%$ und bei $9 \mathrm{~F}$ auf $-i s$ zu $100 \%$.

${ }^{13}$ Nur für belebte Nomen ist die weitere Entwicklung zur schwachen Flexion möglich, belegt sind oblique Kasusformen wie des Autoren, dem Föten (Harnisch 2002).
} 

-us/-os = starke M,
-o/-um/-on = starke $\mathrm{N}$,
$-a /-i s=$ schwache $\mathrm{F}$,
Globus, Mythos,
Konto, Album, Stadion,
Mensa, Praxis.

Fehlt ein solches Suffix, tritt das reguläre Pluralsuffix auf: Diskont - Diskonte.

\subsubsection{Anglizismen und Gallizismen}

Auch für Anglizismen und Gallizismen wird der s-Plural von deutschen Sprechern nur unter bestimmten Bedingungen ausgebildet, wie sein Nicht-Auftreten an Entlehnungen aus diesen Sprachen zeigt (die *Gangsters, *Faxes, *Ingenieurs). Auch hier stellt der $s$-Plural nur eine Übergangslösung dar, wie die Entwicklungen in (33) belegen, so dass sich auch hier die Frage stellt, warum nicht gleich Balkone etc. gebildet wird?

(33) die Fracks > Fräcke, Balkons > Balkone, Lifts > Lifte, Vibrations > Vibrationen, Scheichs > Scheiche, Plans > Pläne, Parks > Parke etc

Bei morphologisch einfachen oder als einfach klassifizierten Fremdwörtern, Entlehnungen aus dem Englischen und Französischen, liegt in jedem Fall Grundformflexion vor, in einem Fall wird an den Singularstamm ein $-s$, im anderen Fall ein - $e$ oder - en angefügt. Die Motivation für das $s$-Suffix liegt auch hier darin, dass mit diesem Suffix im Gegensatz zu den nativen silbischen Suffixen die Identität zwischen Singular und Plural besser gewahrt wird, auch wenn hier keine Endung vor dem Kappen zu bewahren ist, sondern "nur" die Prosodie und die Syllabierung betroffen sind. Die $s$-Formen haben hier gegenüber den später ausgebildeten $e$ len-Formen den Vorteil, dass sie singular-ähnlicher, mit anderen Worten strukturbewahrend sind, denn mit dem $s$-Suffix sind Pluralformen möglich, die ihrer korrespondierenden Singularform maximal ähnlich sind, weil -s dank seiner niedrigen Sonorität in den Stamm integriert wird:

(34) $[($ Park $)]-[($ Park $)(\mathrm{s})]$.

Bei der Pluralbildung mittels eines silbischen Suffixes wird dagegen die phonologische Gestalt des Singularstamms mehr oder weniger stark verändert, so dass native Pluralformen vom Singular abweichen können. Auf durch den Umlaut ausgelöste Veränderungen gehe ich hier nicht ein (s. Wegener 2002), denn selbst bei regulärer Pluralbildung können bis zu drei Veränderungen auftreten: Hunde unterscheidet sich von Hund

1. durch die zusätzliche Silbe, also in der prosodischen Struktur, dem Fuß,

2. durch Resyllabierung, die Morphem- und Silbengrenze trennt,

3. durch die Sonorität des stammfinalen Endkonsonanten, s. (36).

(35) Hund - Hun.de

Diese Veränderungen verletzen das Natürlichkeitsprinzip der Transparenz, das Uniformität des Stammes der beiden paradigmatisch zusammenhängenden Formen verlangt. Dies Prinzip duldet keine Veränderungen, es präferiert Formen, die leicht zu identifizieren sind, bei denen keine verdunkelnden (morpho)phonologicschen Prozesse auftreten wie Umlaut, Palatalisierung, Sonorisierung, Tilgung von Segmenten oder Resyllabierung, cf. Dressler (1999: 137). Generell gilt "no modification of a phoneme in the stem or in the affix" (Dressler 1987: 102). 
Speziell die Integrität der Silbengrenzen hebt Mayerthaler (1987: 49) hervor: "A form F is said to be transparent if $\mathrm{F}$ is morphosyntactically transparent, the optimum being the coincidence of syllable and formative boundaries." Nach Neef (1998: 247) stellt Transparenz das "Main Principle of Morphology" dar, das er definiert als "Grammatical words are ideally identical with the phonological realization of the base."14 Die Pluralformen des Deutschen differieren in dieser Hinsicht erheblich und bilden dadurch eine Skala von maximal bis minimal singular-ähnlich (abgesehen vom absolut identischen, aber nicht ikonischen 0-Plural):

\begin{tabular}{|c|c|c|c|c|c|}
\hline \multirow{7}{*}{$\begin{array}{l}\text { Sg: } \\
\text { Pl: } \\
\text { Sg: } \\
\text { Pl: }\end{array}$} & Ecke & Frau & Boot & Hund $[\mathrm{t}]$ & Hand $[\mathrm{t}]$ \\
\hline & Ecken & Frau.en & Boo.te & Hun.de [d] & Hän.de [d] \\
\hline & Park & Knie [kni:] & Bank & Burg $[\mathrm{k}]$ & Buch $[\mathrm{x}]$ \\
\hline & Parks & Kni.e [kni.ə] & Ban.ken & Bur.gen $[\mathrm{g}]$ & Bü.cher [ç] \\
\hline & $\begin{array}{l}\text { maximal } \\
\text { ähnlich }\end{array}$ & & & & $\begin{array}{l}\text { minimal } \\
\text { ähnlich }\end{array}$ \\
\hline & Änderung & + Änderung & + Änderung & + Änderung & + Änderung \\
\hline & Silbenrand & Fußstruktur & Silbengrenze & Endkonsonant & Vokal \\
\hline
\end{tabular}

Ikonische Pluralbildung ohne Veränderung des Fußes ist nur mit den nicht-silbischen Flexiven - $n$ und $-s$ möglich. Das erstere kann aber nur auf eine Schwa-Silbe mit finalem Sonoranten folgen, nach Obstruenten wird es automatisch silbisch: *Bankn. Die silbischen Flexive führen notwendigerweise zu Mehrsilbigkeit, also einer Veränderung der Fußstruktur, und meistens zu Resyllabierung. Bei Endkonsonanten, die der Auslautverhärtung unterliegen, wird diese blockiert, so dass ein stimmloser mit einem stimmhaften Konsonanten alterniert.

Selbst die reguläre Pluralform Berge [ber.gə] differiert von ihrem Singular Berg [berk] dreifach: in der Fuß- und der Silbenstruktur sowie im Endkonsonanten des Stammes. Die Pluralform für den Eigennamen, Bergs [berks], bewahrt dagegen die Strukturmerkmale der Ausgangsform (s.u.), ist maximal singular-ähnlich.

Nur beim $s$-Plural liegt die Morphemgrenze innerhalb der Silbengrenze, d.h. das Basismorphem wird nicht auf zwei Silben aufgespalten, cf. (37), wo ich die Silbengrenzen durch runde Klammern, die Morphemgrenzen durch eckige Klammern anzeige:

$$
([\mathrm{Ber})(\mathrm{g}][\mathrm{e}])-([\mathrm{Berg}][\mathrm{s}])
$$

Die folgenden Diagramme zeigen diese Aufspaltung des Stammmorphems auf zwei Silben beim $e$-, aber nicht beim $s$-Plural:

\footnotetext{
${ }^{14}$ In den Termini der Optimalitätstheorie wird das Transparenzgebot in den Korrespondenzbeschränkungen, welche Identität zwischen dem Singularstamm und der Pluralform verlangen (Output-Output-Korrespondenz, s. Prince/Smolensky 1995, Kager 1999), detaillierter ausformuliert, zur Anwendung auf die Pluralformen s. Wegener i.V.
} 


\begin{tabular}{|c|c|c|}
\hline$\underline{\text { Singular }}$ & s-Plural & e-Plural \\
\hline pros. Wort & pros. Wort & pros. Wort \\
\hline & $\mid$ & $\mid$ \\
\hline $\mathrm{F}$ & F & $\mathrm{F}$ \\
\hline $\mid$ & $\mid$ & \\
\hline$\sigma$ & $\sigma$ & $\hat{\sigma}$ \\
\hline$\underset{\text { Berg }}{\mid}$ & $\left.\right|_{\text {Bergs }}$ & $\underset{\text { Ber }}{\mid}$ \\
\hline
\end{tabular}

Im Gegensatz zur regulären Pluralform Berge [ber.gə] bewahrt die Pluralform Bergs [berks] für den Eigennamen also wesentliche Strukturmerkmale der Singularform, deren stimmlosen Endkonsonanten und insbesondere die Einsilbigkeit. Die $s$-Form entspricht eher der typischen Form, dem Schema von Stämmen, wogegen die $e$-Form eher dem Schema von Pluralformen entspricht (Bybee 1988, Köpcke 1993):

(39) Singular- und Pluralschemata nach Köpcke (1993: 88):

\begin{tabular}{lllll}
\multicolumn{1}{c}{ Singular } & & & \multicolumn{2}{c}{ Plural } \\
\hline & & & & \\
Einsilbig- & Mehrsil- & Mehrsil- & Mehrsil- & Mehrsil- \\
keit, final. & bigkeit, & bigkeit, & bigkeit, & bigkeit, \\
Plosiv, der/ & final. -er, & final. $-e$ & final. $-e r$, & finales \\
das-Klasse & der/das- & die-Klasse & die-Klasse & $-(e) n$, die- \\
& Klasse & & & Klasse
\end{tabular}

Der $s$-Plural ist also der Form des Singulars ähnlicher als die $e$-Form, und das ist der Grund, warum er für Nomen, die wegen spezieller semiotischer Merkmale auf strukturbewahrende Formen angewiesen sind, präferiert wird.

Der unassimilierte Plural Parks verfehlt zwar die "kanonische Struktur" des Trochäus (Eisenberg 1991) und verletzt somit das Natürlichkeitsprinzip der optimalen Wortlänge sowie das Präferenzgesetz für Kodas (Vennemann 1988: 21): "A syllable coda is the more preferred a) the smaller the number of speech sounds in the coda, b) the less the Consonantal Strength of its offset..." , denn es hat einen komplexen Endrand. Es ist außerdem nicht eindeutig, sondern identisch mit dem Genitiv Singular und erlaubt keine Dativmarkierung (*den Parksn). Aber als nichtsilbisches Flexiv bewahrt es die Struktur des Singularstamms, es verändert nicht die Fußstruktur und führt nicht zu Resyllabierung. Somit entspricht die Silbenzahl der Pluralform der des Singularstamms.

$s$-Pluralformen sind im Gegensatz zu nativen Pluralformen mit silbischen Suffixen maximal singularähnlich, strukturbewahrend, s. (36). Letztere verändern die phonologische Struktur der Fremdwörter mehr oder weniger stark, cf. Generals vs Generä.le, Job [dзวp] Jobs [d3ops] vs ?Job.be, Kid [t] Kids [ts] vs ?Kid.de, Parks vs Par.ke, Pär.ke. 
Die Pluralbildung mit $-s$ ist weniger aufwändig, ist kostengünstiger als die mit -e/-en: bei Kid, $J o b$ vs Hit, Top, die alle mit stimmlosem Endkonsonanten gesprochen werden, verlangt eine native Pluralisierung die Entscheidung darüber, ob

- der auslautende Konsonant stimmhaft wird oder nicht (?Hitte - ?Kidde),

- der auslautende Konsonant zum Silbengelenk wird oder nicht, was im positiven Fall eine geänderte Orthographie voraussetzt (?Toppe, ?Jobbe),

- das Nomen stark oder schwach flektiert wird: Generäle - Rivalen,

- nur in Dialekten, in denen der Umlautplural noch produktiv ist: ob der Vokal umgelautet wird oder nicht: Pärke (Alemannisch) vs Parke.

Alle diese Probleme stellen sich beim s-Plural nicht. Dessen höhere Singular-Ähnlichkeit erklärt sein Auftreten bei unassimilierten Fremdwörtern, denn hier ist er funktional. Die Veränderungen, die durch silbische Pluralsuffixe ausgelöst werden, könnten Fremdwörter in ihrer Verbreitung gefährden. Solange die Singularformen noch nicht etabliert sind, sind davon abweichende Formen wie Pizzen, Konten, ?Jobbe, ??Jöbbe, ?Kidde, ?Disken, ?Geishen eine Gefahr für ihr Bekanntwerden. Deshalb werden zunächst s-Formen bevorzugt: Pizzas, Kontos, Jobs, Kids, Diskos, Geishas.

$s$-Pluralformen haben also den Vorteil der Strukturbewahrung, und das erklärt, warum sie bei neu entlehnten Fremdwörtern präferiert werden. Andere Klassen, die auf strukturbewahrende Pluralformen angewiesen sind, sind Onomatopoetika und Eigennamen, und auch hier tritt der $s$-Plural regelmäßig auf, hier ist er sogar stabil. Fremdwörter und andere Neologismen sind aber ihrer Natur nach instabil.

Das Prinzip der Strukturbewahrung gilt daher naturgemäß nur für unassimilierte Fremdwörter. Deshalb ist der $s$-Plural bei Fremdwörtern und Neologismen ein Übergangsplural, eine temporäre Lösung. Wenn nämlich Fremdwörter und Neologismen einen gewissen Bekanntheitsgrad erreicht haben und morphologisch analysiert sind, nicht mehr "fremde Wörter" darstellen, können sie sich auch morphologisch assimilieren, d.h. native Pluralformen entwickeln, Formen, bei denen die Natürlichkeitsprinzipien der optimalen Wortlänge und des IPA entscheidend sind, sodass -e für starke, -en für schwache Nomen eintritt.

(40) stark: Balkons, Lifts, Fracks, Scheichs, Kostüms, Parks, Kiosks,

$\rightarrow \quad$ Balkone, Lifte, Fräcke, Scheiche, Kostüme, Parke, Kioske,

schwach: Mensas, Aulas, Saunas, Datschas, Villas, Rivals,

$\rightarrow \quad$ Mensen, Aulen, Saunen, Datschen, Villen, Rivalen.

Wenn daher der $s$-Plural bei Fremdwörtern als Übergangsplural dient, so kommt es zwangsläufig zu Schwankungen und zu Zweifelsfällen. Diese sind eine unvermeidliche Begleiterscheinung des Assimilationsprozesses.

(41) Scheichs - Scheiche, Lifts - Lifte, Balkons - Balkone

Ein Assimilationsprozess kann auch bei Neologismen auftreten, die aus nativen Stämmen gebildet sind, sowie bei Akronymen. Beispiele dafür sind (nach Paul 1917, Curme 1922, Mugdan 1977, Duden 2001):

(42) Tunichtguts - Tunichtgute, LPGs - LPGen, PKWs - PKW- . 
Die Erklärung für den Abbau des $s$-Plurals liegt darin, dass $s$-Pluralformen eine ganze Reihe von Nachteilen haben, die mehr oder weniger gehäuft auftreten:

1. sie sind bei starken Nomina formgleich mit dem Genitiv Sg: des/die Autos (Verletzung des NPs der Uniformität);

2. sie erlauben keine Dativmarkierung: mit den *Autosn (Verletzung des NPs der formalen Markierung inhaltlicher Unterschiede);

3. sie haben häufig komplexe Silbenendränder: Lifts, Kiosks, Parks (Verletzung des Präferenzgesetzes für Kodas),

4. bilden häufig keine Trochäen: Jobs, Parks (Verletzung des NPs der optimalen Wortlänge),

5. haben häufig markierte Endsilben mit gespanntem Vokal in geschlossener Silbe:

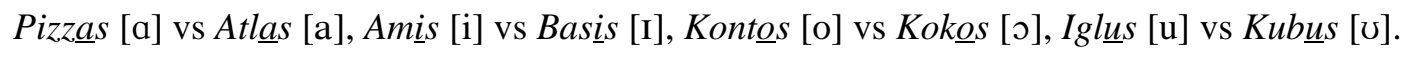

Aus diesen Gründen werden $s$-Formen im Zuge der Assimilation durch assimilierte, d. h. Schwa-Pluralformen ersetzt. Vorübergehend kommt es zwangsläufig zu Zweifelsfällen.

Bei den in quantitativer Hinsicht marginalen, in qualitativer Hinsicht sehr speziellen Onomatopoetika und Eigennamen werden diese Nachteile auf Dauer geduldet, sodass es nicht zu Normproblemen kommt. Die Sprecher wissen sehr genau, wie und wann sie Appellativa oder Eigennamen zu pluralisieren haben. Das gilt sogar für aus Eigennamen entstandene Apellativa. Schwankungen zwischen

\section{(44) die Manns - die Männer, die Kochs - die Köche, die Bäcker - die Beckers die Opels - die Opel, die Diesels - die Diesel, die Dudens - die Duden}

tauchen daher nicht auf. Wenn Fremdwörter in den allgemeinen Wortschatz übergehen und morphologisch assimiliert werden, so stellt der $s$-Plural dagegen nur eine Übergangslösung dar, so dass sich, zunächst als Varianten, assimilierte Pluralformen herausbilden, die sich von nativen höchstens noch orthographisch unterscheiden:

a) Lifte, Scheiche, Fräcke, Pizzen, Villen,

b) Hefte, Reiche, Säcke, Ritzen, Rillen.

Mit den assimilierten Formen in a) liegen genau wie mit den nativen Formen in b) dann trochäische Pluralformen vor, die auf eine sonorant-finale Schwa-Silbe auslauten und damit optimale Pluralformen darstellen, was bedeutet, dass sie die "kanonische Struktur" aufweisen, mit betonter Stammsilbe, die die semantische Bedeutung trägt, und unbetonter Endsilbe, die die grammatische Information trägt, dass sie die Flexionsklasse des Nomens erkennen lassen und bei der starken Flexion die Dativmarkierung ermöglichen.

Bedingung für die Assimilation ist bei morphologisch komplexen Fremdwörtern, dass die Endung als Stammbildungssuffix reanalyiert und dass infolgedessen ihr Kappen toleriert wird. Bedingung für die Assimilation von Anglizismen wie Park ist dagegen, dass die Veränderungen geduldet werden, die durch die Addition einer Silbe ausgelöst werden: Veränderung der Fußstruktur, unter Umständen Verschiebung der Silbengrenze (Par.ke), eventuell Blockierung der Auslautverhärtung des Endkonsonanten (?Jobbe), in Dialekten gar Vokaländerung (Pärke). 
Das Auftreten von Zweifelsfällen ist auch hier nur eine Begleiterscheinung eines natürlichen Sprachwandelprozesses, der Assimilation. Das schließt natürlich nicht aus, dass bei Fremdwörtern, die keine große Verbreitung erlangen und sich nicht im Gemeinwortschatz etablieren können, der Assimilationsprozess unterbleibt. Die Entwicklung ihrer Pluralformen kann beim fremden Plural (Celli, Praktika) oder beim s-Plural (Bars, Schals) stehen bleiben. Die Bedingungen der Integration, zu denen auch das Prestige bestimmter Formen oder die Existenz von Homonymen (Bahren, Schale, Praktiken) gehört, können hier nicht untersucht werden. Es bedarf genauer quantitativer Untersuchungen, um festzustellen, ob die fremde Form oder die hier als Übergangsstufe bezeichnete $s$-Form heute länger besteht als in früheren Zeiten, ob der Assimilationsprozess also heute länger dauert oder ob er gar bei neuen Entlehnungen gänzlich ausbleibt. Die im Gegenwartsdeutschen besonders große Zahl von Anglizismen und die relativ große Vertrautheit deutscher Sprecher mit der englischen Sprache könnte bewirken, dass der Assimilationsprozess seltener erfolgt als früher und schließlich ganz ausbleibt. Zu den Konsequenzen für die deutsche Grammatik s.u.

\subsection{Doppelpluralformen}

In bestimmten Fällen kommt es bei der Assimilation der Fremdwörter zur Ausbildung von Doppelpluralformen. ${ }^{15}$ Dies geschieht zum einen dann, wenn das primäre Pluralsuffix unteranalysiert, als Teil des Stammes interpretiert wird, was bei pluraldominanten Wörtern pragmatisch zu erklären ist: Ihre Referenten tauchen in der Regel eher paarweise oder in Gruppen denn einzeln auf, weshalb die Pluralform hier häufiger als die Singularform vorkommt, also dominant ist. Eine andere Ursache kann darin liegen, dass die primäre Pluralform nicht dem Schema deutscher Pluralformen (s. (39) in 3.2.2) entspricht und durch ein zusätzliches sekundäres Pluralsuffix systematisiert wird. Hier liegt dann Adaptation oder Systematisierung vor.

\subsection{1 -s + -e oder -en}

Die Ausbildung von Doppelpluralformen kommt in zwei entgegengesetzten Fällen vor. Erstens finden wir sie bei Pluralformen auf $-s$, die um ein - $e$, seltener -en, erweitert werden.

Kek-s-e, entlehnt aus dem Englischen cake (Duden 1915: Kek, cf. Köpcke 1993: 149, Fn 85) entwickelt zunächst die Pluralform Kek-s. Eine Generation später wird diese als Singular unteranalysiert, der Duden 1934 vermerkt: Sg Keks, Pl Keks, also Sg = Pl. Zu diesem neuen Singular entwickelt sich nun ein sekundärer Plural, der 1968 noch fakultativ neben der 0-Form steht (Wahrig 1968: Keks-/e), sich in der nächsten Generation bis 1999 aber durchsetzt, Duden 1999: Kekse. Die Fehlanalyse erklärt sich hier dadurch, dass Keks im Deutschen nicht 'Kuchen', sondern 'Kleingebäck' bedeutet, und es sich somit bei Keks um eines der pluraldominanten Wörter handelt. Eine ebensolche Entwicklung und z.T. die selbe Erklärung gilt für

(46) Pumpse, Strapse, Slipse, Chipse, Shrimpse ${ }^{16}$, Kidse (Belege bei Harnisch 2002).

\footnotetext{
15 S. hierzu ausführlicher Wegener (2003).

${ }^{16}$ Hörbeleg: Kellnerin in Potsdam: Wer kriegt die Shrimpse? (Dez. 1999)
} 
Auch hier wird der ursprüngliche $s$-Plural verkannt, die Pluralform als Singular interpretiert und sodann ganz regelgerecht mit -e pluralisiert. ${ }^{17}$

Die Ausbildung des sekundären $e$-Plurals kann jedoch nicht immer auf Unteranalyse des $s$ Suffixes zurückgeführt werden. Wenn die $s$-Formen nicht als Singularformen verwendet werden (?ein Slips, ?ein Chips, ?ein Kids), stellen die e-Formen Fälle von Adaptation dar (s. Plank 1981: 77, Haspelmath 1993: 305, van Marle 1993), d.h. die Sprecher beurteilen die Pluralform als schlecht und "verbessern" sie durch Addition eines Schwas, nehmen eine systemkonforme Systematisierung vor.

Das Ergebnis der Fehlanalyse oder Adaptation der s-Plurale sind nativähnliche, flexionsklassenspezifische Pluralformen, im allgemeinen solche der starken Flexion auf $-e$. In folgendem Beleg liegt jedoch ein schwaches Maskulinum vor:

(47) die Rep-s-en (Rep = Kurzwort für Republikaner)

Für Jeans, das heute als Singular und als Plural gebraucht wird, also die Stufe von Keks 1934 erreicht hat, ist aufgrund der Flexionsklasse die Form ?Jeansen zu erwarten, was dem auch im Singular gebrauchten Hosen $(\mathrm{Sg}=\mathrm{Pl}$ ) entspräche. Allerdings sind -s-en-Formen selten, kommen aber in der österreichischen Kindersprache vor (Autosen, s. Dressler et al. 2001: 123).

Im Gegensatz zur häufigen Ersetzung von $-s$ durch ein natives Suffix bleibt im besonderen Fall der pluraldominanten Wörter das $-s$ als Teil des Stammes erhalten, zumal Muster unter den nativen Wörtern mit auslautendem $-s$ vorliegen (einfache wie Riss, Kuss sowie verkappte $s$-Plurale wie Klecks, Knicks, Klops), die einen $e$-Plural bilden.

Bei der Ausbildung dieser Doppelpluralformen erfolgt die Assimilation zwar auf andere Weise als bei der einfachen Ersetzung von $-s$ durch -e/en, aber sie schließt ebenfalls Normprobleme und Zweifelsfälle ein. Diese erstrecken sich hier sogar auf die Singularformen, die zwangsläufig eine Zeit lang zwischen einer Form ohne und einer mit finalem - $s$ schwanken, dazu s.u.

\subsubsection{Fremdes Suffix $+-s$}

Ferner kommt es zu Zweifelsfällen bei Pluralformen mit fremdem Suffix, das nicht als Pluralsuffix erkannt und dann um $-s$ erweitert wird:

(48) Spagett-i-s, Zucchin-i-s, Graffit-i-s, Scamp-i-s, Sol-i-s, Bambin-i-s, Paparazz-i-s, Praktik-a-s, Antibiotik-a-s, Vis-a-s, Kosmetik-a-s, Lexik-a-s, Rom-a-s, Lemma-ta-s (Harnisch 2002), Intern$a-s^{18}$.

Hier kann, muss aber nicht Unteranalyse der Pluralform als Singular vorliegen (ein Spagetti, aber ?ein Praktika), es kann auch pure Adaptation vorliegen, d.h. die Sprecher "verbessern"

\footnotetext{
${ }^{17}$ Interessant ist, dass dieses Flexiv bisweilen im Spracherwerb als Pluralsuffix auftaucht, s. Ramge (1975: 73), dessen Sohn im 3. Lebensjahr mehrere Formen damit bildet: Waggonse, Männerse, Kuchense, Papisse, Fliegerse. Haspelmaths Tochter bildete Flipse (p.M.). Für österreichische Kinder sind die Formen Autosen, Erbsisen, Ballisen belegt, s. Dressler et al. (2001: 123).

${ }^{18}$ Hörbeleg, SFB 19.3.2002, Autor W. Maier, Richter
} 
die Pluralform, nehmen eine systemkonforme Systematisierung vor. Der hier auftretende Affix-Pleonasmus rührt demnach aus der "Tendenz zur Systematisierung nach dem Prinzip 'eine Bedeutung - eine Form'". Wenn auch bei Vorliegen von Flexivalternativen im allgemeinen "die Generalisierung einer der Alternativen und Verlust der anderen" auftritt, ist doch die "gleichzeitige Realisierung der beiden Alternativen" nicht ausgeschlossen (Plank 1981: 77).

Die Formen in (46) zeigen, dass einsilbige Formen auf -s (Schrimps, Keks) keine guten Pluralformen darstellen, dem Pluralschema mit der kanonischen Zweisilbigkeit und der finalen Schwa-Silbe zu wenig entsprechen. Die letzteren Formen zeigen dagegen, dass bei mehrsilbigen Formen wie Spaghetti, Graffiti im Sprecherbewusstsein ein finales -s eine bessere Pluralmarkierung darstellt als das fremde Suffix. Insofern stellen mehrsilbige s-Pluralformen eventuell ein weiteres Pluralschema des Deutschen dar.

\subsubsection{Durch Pluralformen bedingte Zweifelsfälle bei Singularformen}

In beiden Fällen treten nun parallel zur Unteranalyse des Pluralsuffixes als Teil des Stamms Unsicherheiten über die Formen des Singulars auf.

(49) Wer will den letzten?Schrimp/Schrimps?

Wo ist der andere?Pump/Pumps?

Dir ist ein ?Spagettil?Spagetto auf die Hose gefallen.

Bei Keks, Straps, Klecks, Klaps, Klops ist das $-s$ inzwischen fest zum Bestandteil des Stammes geworden, bei Chip, Schrimp, Kid ${ }^{19}$ ist die Frage offen. Eisenberg (1998: 231) betrachtet Formen wie Chips als Pluralia tantum. Da sie aber in Singularkontexten sehr wohl verwendet werden können (siehe die Beispiele oben), handelt es sich hier um Pluralformen, die identisch mit Singularformen sind.

Zweifelsfälle bei Singularformen, die unabhängig von der Pluralbildung bestehen, tauchen bei Singularstämmen und Ableitungen auf, die auf einen Vollvokal auslauten. Da die unmarkierte Struktur des Deutschen verlangt, dass ein mehrsilbiges Wort auf eine Schwa-Silbe auslautet, kann es auch hier zur Assimilation kommen, was bedeutet, dass der Vollvokal zum Schwa reduziert wird. Dies ist in einigen wenigen Fällen sogar bei $i$-Ableitungen erfolgt, die das für Feminina typische Muster ${ }^{20}$ annahmen, was bei Taxi - Taxe einen Genuswechsel impliziert.

$$
\text { Datscha - Datsche, Taxi - Taxe, Tussi - Tusse. }
$$

\subsection{Ausbleiben von Schwankungen}

Die Funktionalität des $s$-Plurals wird durch die Tatsache bestätigt, dass es bei bestimmten Fremdwörtern gar nicht zu Zweifelsfällen kommt, weil sie direkt integriert werden, so dass hier keine Zwischenformen ausgebildet werden. Wie die Beispiele zeigen, geschieht dies re-

\footnotetext{
${ }^{19}$ Meibauer (2002: 21) führt als Beispiel für Entlehnungen die Nomen Kids, Airbag an.

${ }^{20}$ Das Muster ist außerdem typisch für schwache Maskulina, aber nur, sofern Belebtheit vorliegt.
} 
gelmäßig in zwei Fällen, bei Nomen, die auf einen Sibilanten, und bei solchen, die auf eine Schwa-Silbe auslauten: ${ }^{21}$

(51a) fremder Plural

boxes

chances

bosses

image

(51b) gangsters

computers

examina

nomina

genres

oranges
s-Plural

*Boxes

*Chances

*Bossses

?Images $^{22}$

*Gangsters

*Computers

*Examens

*Nomens

? Genres $^{23}$

*Oranges assimilierter Plural

Boxen

Chancen

Bosse

Image

Gangster-

Computer-

Examen-

Nomen-

Genre-

Orangen

In diesen Fällen gelingt die Entwicklung einer assimilierten Pluralform sozusagen in einem Sprung. Dies kann bei den auf einen Sibilanten auslautenden Nomen phonologisch durch das Geminatenverbot erklärt werden (*Faxs). Denn hier müsste in jedem Fall wie im Englischen ein silbischer Plural gebildet werden, der aber gegenüber den nativen Schwa-Pluralen nur Nachteile hätte (er wäre formgleich mit dem Genitiv Singular und ließe keine Dativmarkierung zu: des Bosses/ die *Bosses, *den Bossesn). Letzteres gilt auch für die auf eine SchwaSilbe auslautenden Nomen (des Gangsters / die *Gangsters, den *Gangstersn, den *Orangesn). Sie sind mithilfe des 0- bzw. n-Plurals, nicht-silbischen Suffixen also, leichter und vollständiger zu assimilieren als mit dem $s$-Suffix.

Fremdwörter mit finaler Schwa-Silbe verhalten sich allerdings anders, wenn sie auf -el auslauten. Diese können im Gegensatz zu denen auf -e, -er oder -en einen $s$-Plural bilden:

(52) die Models [modəls], die Singles ${ }^{24}$, Levels

Es ist im Allgemeinen schwer zu sagen, ob diese Fremdwörter

- eine Ausnahme darstellen, da sie im Gegensatz zu allen anderen Nomen mit Pseudosuffix den Plural mit $-s$ bilden, oder ob sie

- keine Ausnahme darstellen, da die Endung -el die einzige der fraglichen Schwa-Silben ist, die das Pluralschema des Deutschen nicht erfüllt. Nomen auf - $e l$ weisen im Gegensatz zu den anderen nicht schon im Singular eine typische Pluralform auf und bedürfen daher offensichtlich eher einer overten Pluralmarkierung.

Der nicht-ikonische Nullplural wird auch bei nativen Nomen auf -el, aber kaum bei solchen auf -er und -en (außer in der Kindersprache) "repariert", wie in 2.3 gezeigt wurde (Bengels,

\footnotetext{
21 Zimmer (1997: 58) bezeichnet es als "die pure Willkür", dass für Notebook der Plural mit -s, für User aber ohne $-s$ gebildet wird. Tatsächlich sind beide Formen völlig regulär.

22 Duden 2001 gibt zwar Images an, aber Gerhard Schröder sagte im August 2002 "es geht hier nicht um Image".

23 Auch hier gibt Duden 2001 Genres an.

${ }^{24}$ Bei Single hängt der Plural nach Duden 2001 vom Genus und der Bedeutung ab, für den oder die Single (auch die CD) mit -s, das Single ( = 'Match') ohne.
} 
Madeln). Der Nullplural wird hier also eher als bei den anderen Nomen als Fehlen des Pluralsuffixes interpretiert. Der Grund dafür dürfte sein, dass finales -el im Gegensatz zu - e, -er und -en keinem Pluralsuffix entspricht, cf. die einmorphemigen Formen in (53a) mit den zweimorphemigen in (53b):

$\begin{array}{lc}\text { (53a) Eimer, Gangster } & \text { b) Ei-er, Biest-er } \\ \text { Balken, Nomen } & \text { Bank-en, Uhr-en } \\ \text { Käse, Gebirge } & \text { Ross-e, Berg-e } \\ \text { Enkel, Model } & --\end{array}$

Bei den Formen auf -e, -er oder -en wird offenbar der Wortauslaut mit einem der Pluralsuffixe identifiziert, so dass die auslautende Schwa-Silbe eine doppelte Funktion erfüllt, auch wenn dies der Kompositionalität widerspricht: sie ist Wortauslaut und Pluralsuffix zugleich. Das ist bei -el nicht möglich.

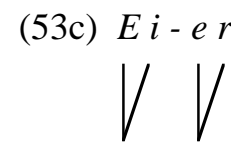

St $\quad \mathrm{Pl}$

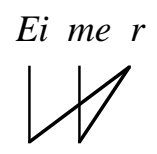

St Pl

Insofern verhalten sie sich ähnlich wie die unflektierbaren Stadtadjektive, die als flektiert interpretiert werden (s. hierzu Fuhrhop 2003): Bei Berlin-er liegt ein Ableitungssuffix vor, das sowohl die Ableitung schließt als auch den Genitiv Plural anzeigt:

(53d) Besuch alter Museen - Besuch Berliner Museen ${ }^{25}$

Besuch a $1 \mathrm{t}$ - e r Museen<smiles>C=CCCCC</smiles>

St GenPl
Besuch Berlin e r Museen

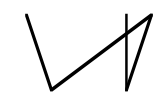

Abl GenPl

Das Bemerkenswerte ist, dass die Pseudosuffixe der Nomen in (53a) im Gegensatz zum Ableitungssuffix - er nicht segmentierbar sind, aber dennoch die Pluralinterpretation der Suffixe in (53b) erhalten können, sogar bei Fremdwörtern. Bei Nomen auf -el erfolgt die Pluralinterpretation aus dem Kontext, der deshalb eindeutig sein muss und den 0-Plural bei Feminina ausschließt (ich habe (die/zwei) Engel, *Ampel gesehen) und gelingt bei etablierten Fremdwörtern ebenso: Onkel, Möbel.

\section{Die Bedeutung der Zweifelsfälle für die Grammatik des Deutschen}

Es bedarf keiner besonderen Erörterung, dass durch den Übergang von irregulären zu regulären Formen und also den Abbau von markierten Klassen die Grammatik einer Sprache einfacher wird. So gesehen sind die in Kapitel 2 beschriebenen Normprobleme eigentlich zu begrüßen. Es kann angenommen werden, dass er- und Umlautplural bis auf wenige hochfrequente Formen, die dann als Ausnahmen weiterleben, aussterben, dass wir also im Deutschen ähnlich wie im Englischen nur noch Relikte bewahren werden: Kinder, Füße, Mäuse, sicher auch noch Hände, Mütter, Töchter, Brüder, Väter, aber man beachte, dass das

25 Die Berlin-Card berechtigt "zum Besuch Berliner Museen". 
Lehnwort Onkel, obwohl Verwandtschaftsbezeichnung, im Gegensatz zu allen nativen Nomen dieser Klasse keinen Umlautplural ausbildete. Für Maskulina mit belebten Referenten besteht hier außerdem die Möglichkeit der schwachen Flexion, die die süddeutsche Form Onkeln zu wählen scheint.

Die durch die Resemantisierung der schwachen Maskulina bewirkten Veränderungen machen das System ebenfalls einfacher, da natürlicher, denn diese Klasse wird jetzt stärker außermorphologisch motiviert.

Dagegen ist es im Allgemeinen eine offene Frage, wie stark die für native Wörter geltenden morphologischen Strukturbildungsprinzipien und Beschränkungen sind, um für Fremdwörter grundsätzlich einen Assimilationsprozess einzuleiten und zu Ende zu führen. Besonders von journalistischer Seite werden angesichts der großen Zahl von Anglizismen Zweifel an der Assimilations- und Integrationsfähigkeit der deutsche Sprache laut (Zimmer 1997: 74).

$\mathrm{Zu}$ bedenken ist hier aber, dass Anglizismen und Gallizismen, sofern sie Flexionsklassenspezifische Pluralformen ausbilden, vollständiger in das deutsche Flexionssystem integriert werden als die Entlehnungen aus den klassischen Sprachen und dem Italienischen, die zum einheitlichen en-Plural der Stammflexion übergehen. Die ersteren "verhalten sich vergleichsweise harmlos und rufen im Gesamtsystem der Substantivflexion kaum Störungen hervor" (Eisenberg 2000: 21), die letzteren dagegen etablieren ein Subsystem, indem die Flexionsklassenspezifik der Pluralformen abgebaut und damit ein wesentliches Prinzip der deutschen Substantivflexion, das des implikativen Paradigmenaufbaus, aufgegeben wird.

Es kann jedoch nicht ausgeschlossen werden, dass der Zeitpunkt erreicht ist, wo der Assimilationsprozess bei Entlehnungen aus dem Englischen unterbleibt. Die im Gegenwartsdeutschen besonders große Zahl von Anglizismen, deren i.a. hohes Prestige und die relativ große Vertrautheit deutscher Sprecher mit der englischen Sprache könnte bewirken, dass der Assimilationsprozess heute länger dauert und seltener erfolgt als früher, oder gar, dass er ganz ausbleibt. Das würde bedeuten, dass es gar nicht mehr zu Zweifelsfällen und Normproblemen bei der Pluralbildung fremder Substantive kommt. Dass dies nicht nur Vorteile hätte, wurde in 3.2 gezeigt. Das wirft die Frage auf, welche Bedeutung die Fremdwörter für die Grammatik des Deutschen haben, die hier nur ansatzweise diskutiert werden kann.

Bestehen zu einem bestimmten Singularstamm zeitlich nacheinander mehrere Pluralformen, so handelt es sich um diachrone Veränderungen in einem Minisystem. Für die nacheinander bestehenden unterschiedlichen Formen der Fremdwörter und die unidirektional verlaufenden Veränderungen sind mehrere Erklärungen möglich.

Nimmt man an, dass die fremden Formen die Beschränkungen der Zielsprache nur unvollkommen erfüllen, so wären die Veränderungen dadurch zu erklären, dass sich die Formen ändern, um die im Deutschen gültigen Beschränkungen zu erfüllen. Danach wäre der Assimilationsprozess ein asymmetrischer Prozess, bei dem die Fremdwörter ans System der deutschen Pluralbildung angepasst werden, dieses sich aber selbst nicht ändert (s. Eisenberg 2001). Also beruhte die Fremdwort-Assimilation nur auf Anpassungsdruck. Für die Anglizismen und Gallizismen könnte diese Erklärung ausreichen. Deren $s$-Plurale wären dann aus 
der Quellsprache mitentlehnt und würden im Zuge ihrer Assimilation ans Deutsche aufgegeben.

Wie gezeigt, können die $s$-Plurale der anderen Fremdwörter so jedoch nicht erkärt werden, werden sie doch erst im deutschen Sprachgebiet ausgebildet. Pizzas, Kontos gibt es nicht im Italienischen, Themas nicht im Griechischen, Generals nicht im Französischen. Das spricht dafür, dass mindestens zwei unterschiedliche Regelsysteme anzunehmen sind, die für die Pluralformen auf den verschiedenen Stufen der Assimilation gelten. Unassimilierte Fremdwörter haben demnach eine eigene Grammatik, die von Prinzipien bestimmt wird, die für die nativen Wörter nicht oder weniger gelten. Für die unassimilierten Fremdwörter konnte gezeigt werden, dass für sie ein Prinzip der Strukturbewahrung Vorrang hat. Mit ihrem s-Plural stellen sie innerhalb der deutschen Nominalflexion, deren Formen i.a. die Prinzipien des konstruktionellen Ikonismus, des IPA, der optimalen Wortlänge und der Uniformität gegenüber dem der Transparenz präferieren, einen Sonderfall dar, der durch ein sonst eher dispräferiertes Natürlichkeitsprinzip zustande kommt.

Nach dieser Annahme stellt die Fremdwortassimilation einen teilweise symmetrischen Prozess dar, bei dem die Fremdwörter zwar integriert werden, aber nicht vollständig. Sie werden demnach nicht einfach an die Beschränkungen der Grammatik der Nehmersprache angepasst, sondern behalten Teile ihrer Eigenschaften, so dass sie innerhalb der Zielsprache, genauer an deren Peripherie ein oder mehrere Epizentren aufbauen, deren Beschränkungen sich nur teilweise mit denen der Kerngrammatik decken. Es ist unter einer solchen Annahme nicht nötig anzunehmen, dass für die nativen Formen nur eine Kerngrammatik existiert - die nativen Formen können selbst schon mehrere Schichten mit unterschiedlichen Beschränkungen bilden.

Unassimilierte Fremdwörter stellen einen Teilbereich des Wortschatzes an der Peripherie dar, für den z.T. andere Prinzipien und Regeln gelten als für den Kernwortschatz und den Systembereich. Dieser periphere Bereich besteht im Deutschen nicht nur aus Fremdwörtern, sondern zu ihm gehören noch einige andere Klassen markierter Nomen. Diese speziellen Nomen lassen sich nach zwei Kriterien definieren und in 4 Wortklassen zusammenfassen. Die Kriterien sind ein phonologisches (K1) und ein pragmatisches (K2):

(54) K1: das Nomen lautet auf einen unbetonten Vollvokal aus,

K2: das Nomen hat aufgrund seiner Funktion oder Neuheit einen speziellen semiotischen Status und ist deshalb auf transparente Flexionsformen angewiesen.

"Spezielle Nomen" mit $s$-Plural sind danach

(55) 1. auf unbetonten Vollvokal auslautende Wörter: die Opas, Unis, Kinos,

2. konsonantisch auslautende Fremdwörter u.a. Neologismen (einschließlich Akronyme, Zusammenrückungen, Substantivierungen): die Balkons, Laptops, Loks, Hochs, Stelldicheins,

3. Onomatopoetika und zitierte Wörter (metalinguistische Substantivierungen): die Kuckucks, die "Ach"s und "Weh"s, ${ }^{26}$

4. Eigennamen: die zwei Berlins, die Manns, die (Opel)Kadetts.

${ }^{26}$ Diese werden im folgenden zusammengefasst als Onomatopoetika. 
Es ist weder ein Zufall noch eine idiosynkratische Eigenschaft des Deutschen, dass genau diese Wortklassen sich phonologisch und morphologisch speziell verhalten, eine besondere Grammatik entwickeln. Uspensky und Zhivov (1977) zeigen, dass dies in vielen Sprachen so ist: "Loanwords, interjections, onomatopoeic forms, ideophones, and nursery words can be identified by the feature of their belonging to another system" (ibd. 1977: 8), ${ }^{27}$ woraus die Autoren das Universal formulieren: "For any language, in a given (peripheral) class there exists a formally anomalous element" (ibd. 1977: 9). Sie zeigen weiter, dass die peripheren Schichten der Sprache deshalb nicht regellos sind: "the chaos and inconsistency of the periphery of language have been greatly exaggerated and (...) we can assume it to be governed by definite principles." (ibd. 1977: 18) Es ist deshalb nicht nötig, für den s-Plural den Status eines Defaults anzunehmen (s. Marcus et al. 1995). Es zeigt sich vielmehr, dass Fremdwörter zusammen mit anderen speziellen Wörtern mindestens eine besondere Schicht des Lexikons an der Peripherie mit eigener Grammatik bilden.

Wie viele Schichten lassen sich hinsichtlich des Parameters Pluralbildung nun im deutschen Wortschatz feststellen? $\mathrm{Zu}$ bedenken ist, dass auch der native Wortschatz keine absolut homogene, durch eine einfache Regel organisierte Schicht ist. Köpcke (1982: 43f) nimmt eine Aufspaltung des Lexikons in Kern, System und Peripherie an. Danach und nach weiterer Differenzierung der Peripherie ergeben sich für die Pluralbildung insgesamt sechs Schichten (s. hierzu auch Munske 1988: 60):28

1. Den Kern bilden die Umlautplurale und die er-Plurale, sie erreichen dank des Umlauts größte Salienz, maximale Differenz zwischen Singular und Plural und damit größte Eindeutigkeit.

2. Die große Klasse der (e)- und (e)n-Plurale bilden das System, dessen Pluralbildung durch die in Kapitel 2 beschriebenen Regeln und Prinzipien organisiert ist.

3. Die Stammflexionsplurale auf -en entsprechen den Systemformen nur in prosodischer Hinsicht, sind wohlgeformt, verstoßen aber gegen das Natürlichkeitsprinzip des IPA. Sie stehen daher zwischen System und Peripherie.

4.-5. Die erste Schicht der Peripherie bilden die $s$-Plurale, wobei die trochäischen, zu i.a. vollvokalisch auslautenden Stämmen gebildeten Formen wie Muttis dem System eher entsprechen als die nicht trochäischen, zu Einsilbern gebildeten wie Parks. Die ersteren werden auch zu nativen Stämmen gebildet (Uhus) und treten auch als Doppelpluralformen auf (Spagettis), die

\footnotetext{
27 Als Beispiel wird angeführt, dass im Russischen, das Nomen i.a. dekliniert, Indeklinabilia stets Lehnwörter seien. Ähnlich erhalten im Birom, einer nigerianischen Sprache, Lehnwörter kein Klassenpräfix. Dasselbe gilt für andere afrikanische Sprachen, s. Heine (1982: 199), zum Bowili, einer Sprache in Ost-Ghana. Auch im Bantu werden Lehnwörter in eine Null-Präfix-Klasse integriert, m.a.W. in die Klasse mit dem transparentesten Klassenmarker, s. Ibrahim (1973: 65).

${ }^{28}$ Munske (1988: 60f) teilt die Fremdwort-Pluralformen in vier Gruppen ein:

1. assimilierte Formen mit - e oder -en (selten -er, -ien), z.T. mit Umlaut,

2. $s$-Pluralformen,

3. Stammflexionsformen auf -en,

4. Formen mit fremden Pluralsuffixen.
} 
letzteren verletzen die Wohlgeformtheitsbeschränkungen stärker und sind deshalb vermutlich diejenigen, die am stärksten zum Abbau tendieren.

6. Ganz außen stehen die fremden Plurale wie Celli, Antibiotika, die ohne Kenntnis der Gebersprache für deutsche Sprecher unregelmäßig sind.

Das folgende Diagramm, in dem die Kerndomäne am linken Pol erscheint, deckt sich weitgehend aber nicht vollständig mit dem Token-Type-Quotient der Pluralklassen, den ich aus den Angaben von Pavlov (1995: 45-48) zu Type- und Tokenfrequenzen errechnet habe, siehe Tabelle 1 und $2 \mathrm{im}$ Anhang.

Token:Type-Quotient der Pluralklassen:

\begin{tabular}{|c|c|c|c|c|c|c|}
\hline -er & -"(e) & -(e) & $-(\mathrm{e}) n$ & irr. -en & $-s$ & frd \\
\hline 10,14 & 3,94 & 0,91 & 0,82 & 2,29 & 0,17 & 0,1 \\
\hline
\end{tabular}

Der ursprünglich fremde $s$-Plural, der bei den Grammatikern des 18. Jahrhunderts noch keine Rolle spielt (Schmidt-Wilpert 1980), ist jedenfalls heute kein Fremdwort-Plural mehr, sondern der reguläre Pluralmarker für semiotisch oder phonologisch markierte, spezielle Wörter. Konsequent wechselte das alte native Onomatopoetikon $U h u$ denn auch vom $e$-zum $s$-Plural, Kuckuck schwankt zwischen -e und -s. Die Existenz einer weiteren Pluralklasse hat zweifellos die Grammatik des Deutschen verändert, s. (11).

Denkbar ist schließlich auch, dass das Deutsche sein Pluralsystem unter dem Einfluss der Fremdwörter ändert, dass also kontaktbedingter Sprachwandel stattfindet, indem eine weitere Pluralklasse geschaffen wird. Die konkurrierenden Formen in (23) und (24) wären dann eher Beispiele für Schwankungen als für systematische Übergänge in eine Richtung. In diesem Fall würde sich die Grammatik des Deutschen ändern. Diese Annahme impliziert ebenfalls einen asymmetrischen Prozess, allerdings in umgekehrter Richtung: hier würde das System der nativen Pluralbildung an die fremden Formen angepasst oder zumindest unter deren Einfluss verändert. Diese bei Sprachkritikern verbreitete, meist negativ bewertete Vorstellung sieht im Assimilationsprozess einen durch Fremdeinfluss bedingten Sprachwandel.

Für diese Annahme scheint nicht nur die Ausbildung von Doppelpluralformen auf $-s$ zu sprechen (s. 3.3.2), sondern auch die Tatsache, dass der ursprünglich fremde $s$-Plural auch bei zu nativen Stämmen gebildeten Wörtern auftritt, die keine Onomatopoetika oder Eigennamen sind. Diese sollen abschließend hier kurz erwähnt werden.

\subsection{Schwanken der Pluralform bei i-Ableitungen}

Seit relativ kurzer Zeit häufen sich im Deutschen Ableitungen und Kurzwörter, die auf Vollvokal enden: Uni, Tussi, Kino, Limo. Das ist ungewöhnlich, denn bei nativen Wörtern des Zentralwortschatzes ist in dieser Position nur Schwa möglich. Ausnahmen sind nicht zufällig Onomatopoetika und Eigennamen (Uhu, Helga, Uli). Der finale Vollvokal führt bei diesen 
Wörtern wie bei den Italianismen phonologisch bedingt, also notwendigerweise zum s-Plural, um einen Hiat zu vermeiden: die Unis, Tussis, Kinos, Limos.

Eine Art Assimilationsprozess ist auch bei einigen $i$-Ableitungen mit nativen und fremden Stämmen festzustellen, die den $s$-Plural abbauen und durch -en ersetzen, obwohl er hier stabil sein müsste, da ein Ableitungssuffix eigentlich nicht getilgt werden kann. Aber selbst hier sind Übergänge zum en-Plural zu beobachten, obwohl dann der Endvokal getilgt werden muss:

(57) die Sozis > Sozen ${ }^{29}$, die Tussis > Tussen.

Der Sog in Richtung Schwaplural ist hier also stärker als die Klassifizierung des finalen Vollvokals als Ableitungssuffix, das stattdessen wie ein Stammbildungssuffix behandelt und getilgt wird.

Ein Indiz dafür, dass sich die Grammatik des Deutschen unter Fremdeinfluss verändert (hat), ist daher weniger in der Tatsache zu sehen, dass der "Fremdwortplural" $-s$ auch für die nativen Stämme gebraucht wird, denn dies ist phonologisch bedingt, als vielmehr in der Tatsache, dass aus nativen und fremden Stämmen Ableitungen mit finalem Vollvokal und also fremd klingenden Suffixen gebildet werden. Zwar hat es auf $-i$ auslautende Vornamen in hypokoristischer Funktion seit althochdeutscher Zeit immer gegeben (Greule 1983). Ihr starkes Anwachsen im 20. Jahrhundert ist aber auf den Einfluss von Fremdwörtern aus verschiedenen Sprachen zurückzuführen. Greule (1983: 210) zählt 13 Gebersprachen auf, ${ }^{30}$ aus denen das Deutsche $i$-Bildungen entlehnt hat, dazu kommen noch Völkerbezeichnungen wie Somali, Israeli. Das lässt vermuten, dass $i$-Bildungen ohne den Einfluss der Fremdwörter und den $s$ Plurals vermutlich kein solch produktives Fortbildungsmuster darstellen würden. Sie sind insofern der deutlichste Beweis dafür, dass die Assimilation von Fremdwörtern nicht nur Anpassung und Integration ins bestehende System, sondern auch Veränderung dieses Systems selbst bedeutet, dass also ein symmetrischer Prozess erfolgt. Dabei ist hervorzuheben, dass es keineswegs nur die Anglizismen sind, die Strukturen und Grammatik des Deutschen ändern.

Die zahlreichen Ableitungen auf $-i$ und (wenn auch in geringerer Zahl) auf - $o$ sind für die Frage des Fremdeinflusses ebenso bedeutsam wie der Gebrauch von Lehnwörtern mit finalem Vollvokal, zu denen ja selbst Wörter des Nahbereichs wie Papa, Mama, Oma, Opa gehören. Die Bildung neuer Wörter mit finalem Vollvokal widerspricht der unmarkierten Wortstruktur des Deutschen, denn Substantive lauten im unmarkierten Fall - wenn sie nicht einsilbig sind auf ein Ableitungssuffix oder eine Schwa-Silbe aus. Die vollvokalisch auslautenden stellen folglich eine Wortklasse an der Peripherie dar. Diese phonologisch spezielle Wortklasse stellt

\footnotetext{
29 Derzeit ist ein Schwanken zwischen Sozis und Sozen zu beobachten, wobei das Letztere (laut PNN vom 17.11.1999, TAZ vom 3.12.1999) vor allem in CDU-Kreisen, aber auch von den Grünen gebraucht wird und leicht pejorative Bedeutung hat. Die assimilierten Formen, die keine markierten Endsilben haben, werden dann auch als Erstglieder in Komposita gebraucht: Kontenbewegung, Villenarchitektur, Sozenfresser (Berliner Zeitung vom 27.05.2003 über einen CDU-Politiker).

30 Dies sind Englisch (Baby), Französisch (Dementi), Italienisch (Spagetti), Latein (Juni), Indisch (Sari), Arabisch (Mufti), Japanisch (Harakiri), Spanisch (Mahagoni), Polnisch (Strachuli), Hebräisch (Rabbi), Türkisch oder Persisch (Spahi), Persisch-Indisch (Khaki) und eine afrikanische Sprache (Safari).
} 
aber nun sogar eine Optimierung von Lautgestalten dar (Ronneberger-Sibold 1995). Mit der seit dem Ahd erfolgten Reduktion sämtlicher Endsilbenvokale verschenkt das Deutsche ja die Möglichkeit, in der Endsilbe der Wörter mehr als grammatische Information zu übermitteln. Offenbar sind die Sprachbenutzer mit dem "Schutt" der vielen Schwa in den unbetonten Silben ihres Normalwortschatzes nicht "besonders zufrieden", und es sei bezeichnend, dass sie "wieder unbetonte Vollvokale verwenden, sobald sie frei sind, sich neue Wörter zu schaffen." (ibd. 1995: 43). Es ist also die Zunahme von Wortbildungen mit finalem Vollvokal, die auf eine Änderung der deutschen Wortbildung und Wortstruktur unter dem Einfluss der Fremdwörter hindeutet, nicht primär deren Pluralbildung. Die Tatsache, dass selbst bei einigen Ableitungen Assimilation der Pluralform erfolgt, könnte gerade ein Hinweis darauf sein, dass das Deutsche auch diese Fälle integrieren kann.

\section{Lexikoneinträge}

Wie sehen nun die Lexikoneinträge für die Zweifelsfälle aus? Nach den in 2.1 dargelegten Regeln (11a-b) sind sie bis auf wenige Fälle keineswegs willkürlich, sondern folgen lediglich jeweils zwei miteinander konkurrierenden Regeln, und diese Regeln basieren auf unterschiedlichen Merkmalen für die Flexionsklasse im Lexikoneintrag. Mit anderen Worten schwanken die Zweifelsfälle zwischen zwei Pluralklassen, die durch unterschiedliche Merkmale geregelt sind oder von denen die eine Form irregulär und folglich im Lexikoneintrag gespeichert, die andere regulär nach dem Merkmal zu bilden ist.

Bei den auf Abbau der markierten Klassen beruhenden Zweifelsfällen der starken Nomen in Kapitel 2 besteht derzeit ein Lexikoneintrag mit dem Merkmal +er- bzw. + Umlautplural, daneben einer, der dieses Merkmal nicht mehr hat, der daher regulär den Defaultplural für die starken Nomen selegiert, d.h. -e.

Für die Feminina, deren Plural aufgrund von Flexionsklassenwechsel schwankt, gilt entsprechend, dass ein Lexikoneintrag mit dem Merkmal +stark, ein zweiter mit dem Merkmal + schwach existiert. Aus dem ersten folgt der Umlautplural, aus dem zweiten der (e)n-Plural.

Für den irregulär in der starken Flexionsklasse auftretenden (e)n-Plural kann angenommen werden, dass er bei nativen Wörtern ${ }^{31}$ ebenfalls im Lexikoneintrag vermerkt ist. Die Variante mit regulärem 0-Plural hat dieses Merkmal nicht. Für die nicht-nativen Wörter, die an ihrem Stammbildungssuffix - us, -um etc erkenntbar sind, kann angenommen werden, dass sie dadurch für "+Stammflexion" markiert sind. Aus diesem Merkmal folgt dann der enPluralmarker regulär. Nomen, die trotz eines finalen - us den unmarkierten $e$-Plural bilden (Zirkusse), haben entsprechend kein Merkmal für die Pluralbildung, sind nur als [+stark] klassifiziert. Diese Merkmale sind zusammen mit dem Genus außerdem für die Bildung der Genitiv-Formen und natürlich für die Selektion der Artikelformen nötig.

Der $s$-Plural setzt voraus, dass die Nomen spezielle phonologische oder semiotische Merkmale haben und dadurch zu einer der Wortklassen der Onomatopoetika, Eigennamen oder

\footnotetext{
31 Beispiele sind Auge, Ohr, Staat, Herz, Bett, Hemd, Muskel, Name und die in 2.3 aufgeführten. Für Einsilber ist der en-Plural heute unproduktiv.
} 
unassimilierten Fremdwörter o.a. Neologismen gehören. Diese Merkmale lassen sich unter dem Label "spezielle Nomen" (SN) zusammenfassen. Hat ein Nomen eines dieser besonderen Merkmale, so ist es markiert, der $s$-Plural prädiktabel. Für den Lexikoneintrag gilt folglich: Nomen mit dem Merkmal +SN (= +Eigenname, +Onomatopoetikon, +Fremdwort, +finaler unbetonter Vollvokal) selegieren den s-Plural: Schmidts, Achs, Laptops, Opas. Die Variante hat dieses Merkmal nicht und bildet folglich den regulären Plural ihrer Flexionsklasse.

Die spezielle Regel blockiert die Anwendung der allgemeinen Regel: meine Nachbarn, die $*$ Schmidte, die *Ache, *Laptoppe, *Opae. Fehlt das Merkmal [+SN], so kann der s-Plural nicht auftreten: der Beruf der *Schmieds, die *Schuhs, und es tritt die allgemeine Regel in Kraft, die nur durch die Flexionsklasse restringiert ist und starken Nomen den $e$-, schwachen

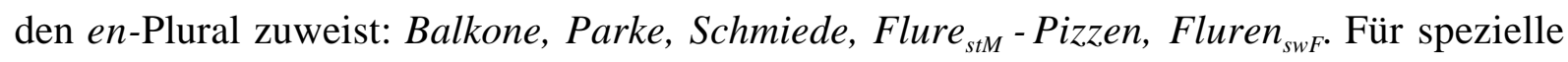
Nomen mit auslautender Schwasilbe oder Sibilant ${ }^{32}$ gilt dann die zusätzliche Restriktion, dass der $s$-Plural nicht appliziert: *Gangsters, *Bosses, *Oranges. Einfacher ist hier allerdings die Lösung, dass solche Nomen aufgrund ihres Auslauts das Merkmal [+SN] nicht erhalten, sondern unmarkiert bleiben, d.h. als für die Pluralbildung unauffällig gelten und damit sofort der allgemeinen Regel zugänglich sind. Die Pluralbildung durch $-s$ oder einen der unmarkierten Pluralmarker -(e) bzw -(e)n stellt also einen Fall der "Elsewhere" oder "Sonst"-Bedingung dar (Kiparsky 1982: 136, s. Wiese 1987: 241f).

Die Übersicht führt einige Beispiele an und zeigt zugleich, dass der Lexikoneintrag und damit die Grammatik in den meisten Zweifelsfällen durch die derzeit zu beobachtenden Veränderungen und Assimilationsprozesse tatsächlich einfacher wird. (Das Merkmal für die Flexionsklasse ist eingeklammert, wenn sich diese aus dem Genus und/oder dem genustypischen Auslaut des Nomens ergibt.)

(58) Beispiel für einige Lexikoneinträge:

\begin{tabular}{|l|l|l|l|l|l|}
\hline & \multicolumn{2}{|c|}{$\begin{array}{l}\text { Merkmal } \\
\text { FK }\end{array}$} & Gen Sg & Pl & Pl-Form \\
\hline Schlot & st, +UPl & M & (e)s & "e & Schlöte \\
\hline Schlot & st & M & (e)s & e & Schlote \\
\hline Schlucht & st & F & - & "e & Schlüchte \\
\hline Schlucht & sw & F & - & en & Schluchten \\
\hline Pizza & FW & F & - & s & Pizzas \\
\hline Pizza & sw & F & - & en & Pizzen \\
\hline Tussi & SN & F & - & -s & Tussis \\
\hline Tussi & (sw) & F & - & en & Tussen \\
\hline
\end{tabular}

\footnotetext{
${ }^{32}$ Für Eigennamen existiert in diesem Fall ein besonderes Pluralsuffix, das die Herkunft aus dem Genitivsuffix belegt: -ens - die Kunz-ens, Grass-ens.
} 


\begin{tabular}{|l|l|l|l|l|l|}
\hline Konto & FW, st & N & s & s & Kontos \\
\hline Konto & SF, (st) & N & s & en & Konten \\
\hline Globus & SF, (st) & M & (s) & en & Globen \\
\hline Globus & (st) & M & (s) & e & Globusse \\
\hline Park & FW, st & M & s & s & Parks \\
\hline Park & st & M & s & e & Parke \\
\hline Shrimp & FW, st & M & s & s & Shrimps \\
\hline Schrimps & st & M & (s) & e & Schrimpse \\
\hline
\end{tabular}

\section{Literaturangaben}

Bornschein, Matthias/Butt, Matthias (1987): "Zum Status des s-Plurals im gegenwärtigen Deutsch." In: Abraham, Werner/Arhammar, Rita (eds.): Linguistik in Deutschland. Tübingen: 135-153.

Bybee, Joan L. (1985): Morphology. A Study of the relation between meaning and form. Amsterdam/Philadelphia.

Bybee, Joan L. (1995): "Regular Morphology and the Lexicon". Language and Cognitive Processes 10: 425-455.

Curme, George O. (1922): A Grammar of the German Language. New York.

Dressler, Wolfgang et al. (2001): "Sources of Markedness in Language Structure". Folia Linguistica Historica 22: 103-135.

Dressler, Wolfang U. (ed.) (1987): Leitmotifs in Natural Morphology. Amsterdam.

Duden: Deutsches Universalwörterbuch, ${ }^{2} 1989,{ }^{4} 2001$.

Eisenberg, Peter (1991): "Syllabische Struktur und Wortakzent: Prinzipien der Prosodik deutscher Wörter". Zeitschrift für Sprachwissenschaft 10: 37-64.

Eisenberg, Peter (1998): Grundriss der deutschen Grammatik, I: Das Wort. Stuttgart.

Eisenberg, Peter (2001): "Die grammatische Integration von Fremdwörtern. Was fängt das Deutsche mit seinen Latinismen und Anglizismen an?". In: Stickel, G. (ed.): Neues und Fremdes im deutschen Wortschatz. Jahrbuch 2000 des IdS. Berlin: 183-209.

Fuhrhop, Nanna (2003): "'Berliner' Luft und 'Potsdamer' Bürgermeister: Zur Grammatik der Stadtadjektive". Lingistische Berichte 193: 91-108.

Harnisch, Rüdiger (1994): "Stammerweiterung im Singular - Stammflexion im Plural. Zum Bautyp der deutschen Substantivdeklination". In: Köpcke, K.M. (ed.): Funktionale Untersuchungen zur deutschen Nominal- und Verbalmorphologie. Tübingen: 97-114.

Harnisch, Rüdiger (2002): "Tendenzen der morphologischen Integration von Fremdwörtern ins Deutsche". In: Wiesinger, Peter (ed.): Akten des 10. Kongresses der IVG. Bd 2. Bern: 73-79.

Kiparsky, Paul (1982): "From cyclic phonology to lexical phonology." In: Hulst, Harry v.d./ Smith, Norval: The structure of Phonological Representation. Dordrecht: 131-175. 
Köpcke, Klaus-Michael (1982): Untersuchungen zum Genussystem der deutschen Gegenwartssprache. Tübingen.

Köpcke, Klaus-Michael (1993): Schemata bei der Pluralbildung im Deutschen. Tübingen.

Köpcke, Klaus-Michael (1994): "Zur Rolle von Schemata bei der Pluralbildung monosyllabischer Maskulina". In: Köpcke, K. M. (ed.): Funktionale Untersuchungen zur deutschen Nominal- und Verbalmorphologie. Tübingen: 81-96.

Köpcke, Klaus-Michael (1995): "Die Klassifikation der schwachen Maskulina in der deutschen Gegenwartssprache". Zeitschrift für Sprachwissenschaft 14: 159-180.

Kluge, Friedrich (1999): Etymologisches Wörterbuch der deutschen Sprache. 23. Aufl. Berlin.

Mackensen, Lutz s.a.: Ursprung der Wörter. Etymologisches Wörterbuch der deutschen Sprache. Wiesbaden.

Meibauer, Jörg (2002): "Lexikon und Morphologie". In: Meibauer, J. et al.: Einführung in die germanistische Linguistik. Stuttgart: 15-69.

Mugdan, Joachim (1977): Flexionsmorphologie und Psycholinguistik. Tübingen.

Munske, Horst Haider (1988): "Ist das Deutsche eine Mischsprache? Zur Stellung der Fremdwörter im Deutschen Sprachsystem". In: Munske, H.H. et al. (eds.): Deutscher Wortschatz. Lexikologische Studien. Berlin: 46-74.

Neef, Martin (1998): "The Reduced syllable plural in German". In: Fabri,R./Ortmann A./ Parodi, T. (eds.): Models of Inflection. Tübingen: 244-265.

Nübling, Damaris (2001): "Zur Entstehung und Struktur ungebändigter Allomorphik: Pluralbildungsverfahren im Luxemburgischen". Vortag auf der Tagung der SLE in Leuven am 29.8.2001.

Paul, Hermann (1917): Deutsche Grammatik. Band II: Flexionslehre. Tübingen.

Pavlov, Vladimir (1995): Die Deklination der Substantive im Deutschen. Synchronie und Diachronie. Frankfurt/M.

Plank, Frans (1981): Morphologische (Ir)Regularitäten. Tübingen.

Ronneberger-Sibold, Elke (1995): "Die Optimierung von Lautgestalten durch Wortkürzung und durch langfristigen Sprachwandel". In: Boretzky, N. et al. (eds.): Beiträge zum internationalen Symposium über "Natürlichkeitstheorie und Sprachwandel"... Bochum: 31-44.

Sachs, Karl (= Sachs-Villatte) (1900): Encyclopädisches Wörterbuch, Bd II: Dt-Frz. Berlin.

Schmidt-Wilpert, Gabriele (1980): "Zur Substantivflexion, Grammatik und Sprachnorm um 1750". Zeitschrift für Deutsche Philologie 99: 410-429.

Thieroff, Rolf (2002): "Was ist markiert? Überleungen zum Plural-Umlaut im Deutschen". Vortrag SLE-Tagung 2002 in Potsdam

Uspensky, B.A./Zhivov, V.M. (1977): "Center-Periphery Opposition and Language Universals". Linguistics 196: 5-24.

Vennemann, Theo (1988): Preference laws for syllable structure and the explanation of sound change. Berlin.

Vennemann, Theo (1991): "Skizze der deutschen Wortprosodie". Zeitschrift für Sprachwissenschaft 10: 86-111.

Wahrig, Gerhard (1975): Deutsches Wörterbuch, Gütersloh/Berlin. 
Wegener, Heide (1995): Die Nominalflexion des Deutschen, verstanden als Lerngegenstand. Tübingen. (= Reihe Germanistische Linguistik 151).

Wegener, Heide (1999): "Die Pluralbildung im Deutschen - ein Versuch im Rahmen der Optimalitätstheorie". Linguistik online 4, 3/1999.

http://www.linguistik-online.de/3_99/wegener.html bzw.

http://www.linguistik-online.de/3_99/wegener.pdf.

Wegener, Heide (2002): "Aufbau von markierten Pluralklassen im Deutschen - eine Herausforderung für die Markiertheitstheorie". Folia Linguistica 36: 261-295.

Wegener, Heide i.V.: "Von Pizzen und Keksen. Zur morphologischen Assimilation der Fremdwörter".

Wiese, Richard (1987): "Phonologie und Morphologie des Umlauts im Deutschen". Zeitschrift für Sprachwissenschaft 6: 227-248.

Wiese, Richard (1996): The phonology of German. Oxford.

Wurzel, Wolfgang U. (1984): Flexionsmorphologie und Natürlichkeit. Ein Beitrag zur morphologischen Theoriebildung. Berlin.

Wurzel, Wolfgang U. (1985): "Deutsch der Funke zu der Funken: ein Fall für die natürliche Morphologie". Linguistische Studien A 127: 129-145.

Wurzel, Wolfgang U. (1987): "System-dependent morphological naturalness in inflection". In: Dressler, W.U. (ed.) (1987): 59-98.

Wurzel, Wolfgang U. (1988): "Zur Erklärbarkeit sprachlichen Wandels". ZPSK 41: 488-510.

Wurzel, Wolfgang U. (1990): "Gedanken zu Suppletion und Natürlichkeit". ZPSK 43: 86-91

Wurzel, Wolfgang U. (1996): "Morphologischer Strukturwandel: Typologische Entwicklungen im Deutschen". In: Lang, Ewald/Zifonun, Gisela (eds.): Deutsch - typologisch. Berlin: 492-524.

Zimmer, Dieter E. (1997): "Neuanglodeutsch. Über die Pidginisierung der Sprache". In: ders.: Deutsch und anders. Die Sprache im Modernisierungsfieber. Hamburg: 7-85. 
Anhang 1: Produktivität der Pluralklassen

Tabelle 1: Statistische Entwicklung der Pluralformen während des 20. Jahrhunderts -

Typefrequenzen $^{33}$ der regulären und irregulären Pluralformen im Standarddeutschen, $\mathrm{N}=$ 2307 (Mugdan 1977:97, nach Kaeding 1898), bzw. N = 6505 (Pavlov 1995:45, nach Wahrig 1975)

\begin{tabular}{|l|c|l|l|l|l|l|}
\hline \multicolumn{4}{|c|}{ Regulär } & \multicolumn{3}{c|}{ Irregulär } \\
\hline & $\begin{array}{l}\text { schwach: } \\
-(\mathrm{e}) \mathrm{n}\end{array}$ & $\begin{array}{l}\text { stark: } \\
-(\mathrm{e})\end{array}$ & $\begin{array}{l}\text { speziell: } \\
\text {-s }\end{array}$ & $\begin{array}{l}\text { gemischt: } \\
\text {-(e)n }\end{array}$ & $\begin{array}{l}\text { stark: } \\
-(\mathrm{e})\end{array}$ & $\begin{array}{l}\text { stark: } \\
\text {-er }\end{array}$ \\
\hline 1898 & 55.78 & 27.35 & 2.6 & 1.04 & 9.23 & 2.3 \\
\hline 1975 & 47.5 & 35.8 & 9.0 & 1.7 & 3.8 & 0.7 \\
\hline$\Sigma \% 1898$ & \multicolumn{3}{|c|}{85.73} & & 12.57 & \\
\hline$\Sigma \% 1975$ & 92.3 & & 6.2 & \\
\hline
\end{tabular}

Tabelle 2: Statistische Entwicklung der Pluralformen während des 20. Jahrhunderts -

Tokenfrequenzen ${ }^{34}$ der regulären und irregulären Pluralformen im Standarddeutschen, N = 376906 (Mugdan 1977:97, errechnet nach Kaeding 1898) bzw. N = 13587 (Pavlov 1995:4548, nach Textkorpus)

\begin{tabular}{|c|c|c|c|c|c|c|}
\hline \multicolumn{4}{|c|}{ Regulär } & \multicolumn{3}{|c|}{ Irregulär } \\
\hline & $\begin{array}{l}\text { schwach: } \\
\text {-(e)n }\end{array}$ & $\begin{array}{l}\text { stark: } \\
\text {-(e) }\end{array}$ & $\begin{array}{l}\text { speziell: } \\
-\mathrm{s}\end{array}$ & $\begin{array}{l}\text { gemischt: } \\
\text {-(e)n }\end{array}$ & $\begin{array}{l}\text { stark: } \\
-"(\mathrm{e})\end{array}$ & $\begin{array}{l}\text { stark: } \\
\text {-er }\end{array}$ \\
\hline 1898 & 50.1 & 25.47 & 0.9 & 5.7 & 14.11 & 3.1 \\
\hline 1975 & 39.4 & 32.8 & 1.56 & 3.9 & 15 & 7.1 \\
\hline$\Sigma \% 1898$ & \multicolumn{3}{|c|}{76.47} & \multicolumn{3}{|c|}{22.9} \\
\hline$\Sigma \% 1975$ & \multicolumn{3}{|c|}{73.8} & \multicolumn{3}{|c|}{26} \\
\hline
\end{tabular}

Tabelle 3: Absolute und relative Produktivität der Pluralklassen -

Veränderung der Prozentanteile der Typefrequenzen der regulären und irregulären Pluralformen im Standarddeutschen während des 20. Jahrhunderts, N = 2307 (Mugdan 1977:97, nach Kaeding 1898), bzw. $N=6505$ (Pavlov 1995:45, nach Wahrig 1975)

\begin{tabular}{|l|l|l|l|l|l|l|}
\hline \multicolumn{4}{|c|}{ Regulär } & \multicolumn{3}{c|}{ Irregulär } \\
\hline & $\begin{array}{l}\text { schwach: } \\
-(\mathrm{e}) \mathrm{n}\end{array}$ & $\begin{array}{l}\text { stark: } \\
-(\mathrm{e})\end{array}$ & $\begin{array}{l}\text { speziell: } \\
-\mathrm{s}\end{array}$ & $\begin{array}{l}\text { gemischt: } \\
-(\mathrm{e}) \mathrm{n}\end{array}$ & $\begin{array}{l}\text { stark: } \\
-"(\mathrm{e})\end{array}$ & $\begin{array}{l}\text { stark: } \\
\text {-er }\end{array}$ \\
\hline absolut & -8.28 & +8.45 & +6.4 & +0.66 & -5.43 & $-1.66 \%$ \\
\hline relativ & -14.85 & +30 & +246 & +63 & -58.83 & $-69.57 \%$ \\
\hline
\end{tabular}

\footnotetext{
33 Abgesehen von "sonstigen" Pluralformen wie Celli für Cello.

34 S. Fn 20.
} 
Anhang 2: Tokenfrequenzen einiger Varianten

Tokenfrequenzen einiger Pluralvarianten, alphabetisch geordnet, nach Auskunft von Internetsuchmaschinen, befragt im März 2003. ${ }^{35}$ Die Werte zeigen meist deutliche Unterschiede zwischen den Varianten, aber bis auf Genale/Generäle und Tussis/Tussen sind die Angaben kohärent hinsichtlich der Frage, welche Form dominiert.

\begin{tabular}{|c|c|c|c|c|}
\hline & Acoon & Altavista & Fireball & Google \\
\hline die Balkons & 85 & 126 & 74 & 227 \\
\hline die Balkone & 3.936 & 1.269 & 645 & 2.160 \\
\hline die Chips & 17.552 & 5.561 & 2.999 & 10.800 \\
\hline die Chipse & 9 & 9 & 3 & 26 \\
\hline die Denkmale & 1.119 & 422 & 190 & 708 \\
\hline die Denkmäler & 5.055 & 1.608 & 829 & 3.210 \\
\hline die Generale & 1.370 & 2.578 & 1.230 & 1.090 \\
\hline die Generäle & 5.594 & 2.063 & 1.426 & 3.710 \\
\hline die Globusse & 1 & 0 & 0 & 4 \\
\hline die Globen & 77 & 127 & 62 & 324 \\
\hline die Keks & 1.936 & 193 & 105 & 375 \\
\hline die Kekse & 7.482 & 2.727 & 900 & 4.860 \\
\hline die Lifts & 25 & 31 & 18 & 98 \\
\hline die Lifte & 2.720 & 924 & 542 & 2.090 \\
\hline die Mensas & 2 & 0 & 0 & 5 \\
\hline die Mensen & 1.930 & 497 & 293 & 1.120 \\
\hline die Parks & 5.866 & 1.882 & 982 & 4.340 \\
\hline die Parke & 36 & 74 & 52 & 117 \\
\hline die Pizzas & 185 & 310 & 172 & 688 \\
\hline die Pizzen & 2.320 & 627 & 269 & 1.290 \\
\hline die Saunas & 11 & 22 & 14 & 53 \\
\hline die Saunen & 122 & 207 & 99 & 566 \\
\hline die Schlote & 1.296 & 450 & 257 & 680 \\
\hline die Schlöte & 10 & 12 & 54 & 34 \\
\hline die Schluchten & 6.143 & 1.886 & 1.216 & 3.990 \\
\hline die Schlüchte & 3 & 3 & 0 & 6 \\
\hline die Shrimps & 1.210 & 491 & 273 & 901 \\
\hline die Shrimpse & 2 & 1 & 0 & 1 \\
\hline die Sozis & 2.735 & 820 & 731 & 2.120 \\
\hline die Sozen & 58 & 95 & 71 & 234 \\
\hline die Tests & 43.141 & 14.296 & 7.289 & 30.400 \\
\hline die Teste & 95 & 158 & 86 & 341 \\
\hline die Tussis & 86 & 124 & 47 & 319 \\
\hline die Tussen & 78 & 68 & 51 & 347 \\
\hline die Villas & 46 & 69 & 40 & 199 \\
\hline die Villen & 4.885 & 1.624 & 959 & 3.960 \\
\hline
\end{tabular}

35 Für diese Idee danke ich Wolf Peter Klein, für die Befragung Nicole Hoffmann. 
Anhang 3: Regionale Unterschiede und Genusunterschiede

Durch einen Test, der mit Studenten der Universitäten Bonn, Potsdam und Zürich durchgeführt wurde, ${ }^{36}$ sollte untersucht werden, ob es 1 . regionale Unterschiede in der Assimilation der Fremdwörter gibt, 2. wie sich das Genus bei gleichem Auslaut auf die Pluralbildung auswirkt. Diagramm 1 zeigt die Ergebnisse für Nomen mit finalem -a:

\begin{tabular}{|c|c|c|c|c|c|c|c|c|c|c|}
\hline \multicolumn{10}{|l|}{ Diagramm 1: Nomen mit Auslaut auf -a } \\
\hline \multicolumn{10}{|c|}{ Bundesland } \\
\hline Auslaut/Genus & NRW & CH & B & BW & H & NS & SA & Bln. & BRB & MVP \\
\hline $\mathrm{a} / \mathrm{M}$ & 28.6 & 0 & 13.8 & 20 & 75 & 20 & 16.7 & 19.4 & 50 & 0 \\
\hline $\mathrm{a} / \mathrm{F}$ & 75.2 & 40.6 & 68 & 68.6 & 89.3 & 67.1 & 66.7 & 68.6 & 78.1 & 90.5 \\
\hline $\mathrm{a} / \mathrm{N}$ & 50 & 53.8 & 65.5 & 70 & 75 & 55 & 66.7 & 65.3 & 82.8 & 83.3 \\
\hline
\end{tabular}

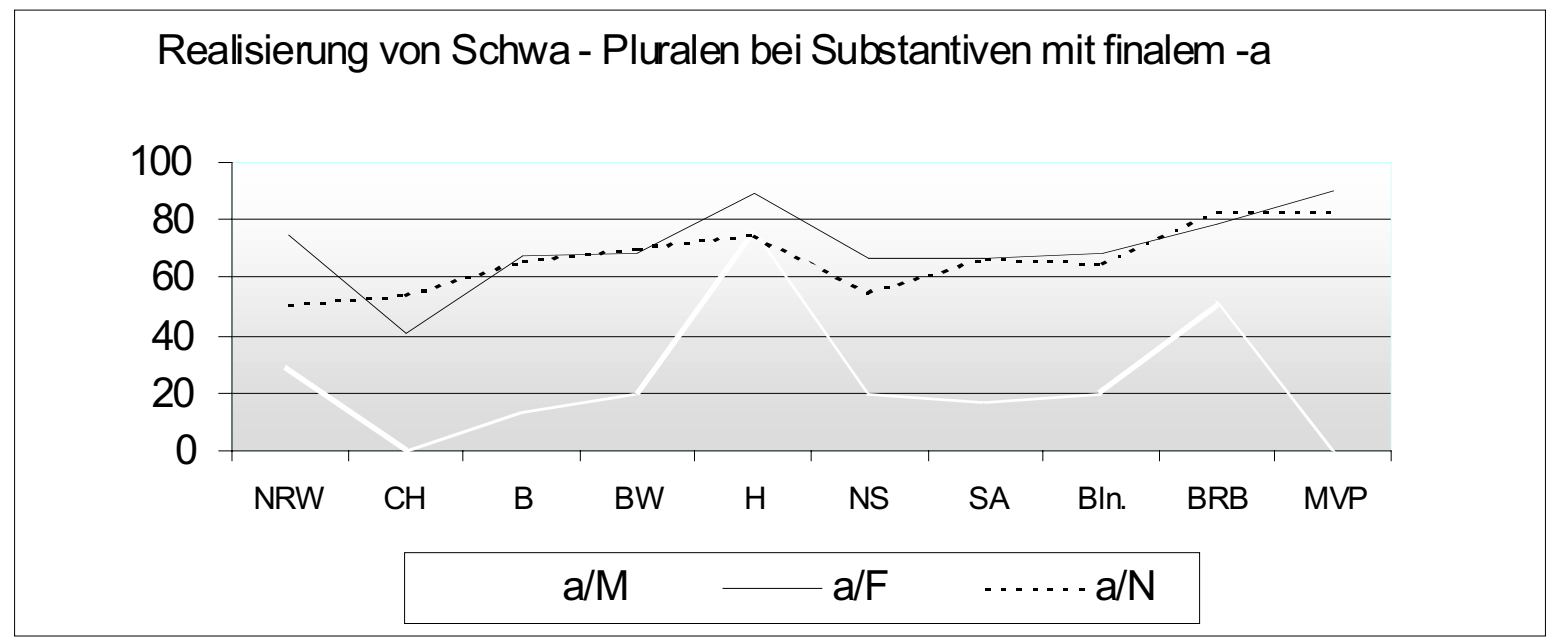

Das Ergebnis zeigt, dass Schweizer bei Nomen auf - $a$ mehr s-Plurale bilden als besonders die Studenten aus NRW, Hessen und Brandenburg, die -en stärker als alle anderen einsetzen.

Alle Probanden bilden besonders viele assimilierte Formen für Feminina auf - $a$, was zu erwarten war, denn hier entspricht diese Form vollständig dem etablierten Muster der Feminina, die zu 96,6\% schwach flektieren (s. Pavlov 1995: 46), s. 3.2.1.

Diagramm 2 zeigt die Ergebnisse für die Zusammenrückungen:

\begin{tabular}{|c|c|c|c|c|c|c|c|c|c|c|}
\hline \multicolumn{11}{|c|}{ Diagramm 2: Zusammenrückungen, maskulin und neutrum } \\
\hline & \multicolumn{10}{|c|}{ Bundesland } \\
\hline & NRW & $\mathrm{CH}$ & $\mathrm{B}$ & BW & $\mathrm{H}$ & NS & SA & Bln. & $\mathrm{BRB}$ & MVP \\
\hline $\mathrm{ZR} / \mathrm{M}$ & 52.4 & 38.5 & 27.6 & 80 & 75 & 70 & 50 & 47.2 & 53.1 & 33.3 \\
\hline $\mathrm{ZR} / \mathrm{N}$ & 10.7 & 11.5 & 10.3 & 20 & 12.5 & 15 & 33.3 & 8.3 & 10.9 & 16.7 \\
\hline
\end{tabular}

\footnotetext{
36 Ich danke Peter Gallmann und Rolf Thieroff für ihre Hilfe.
} 


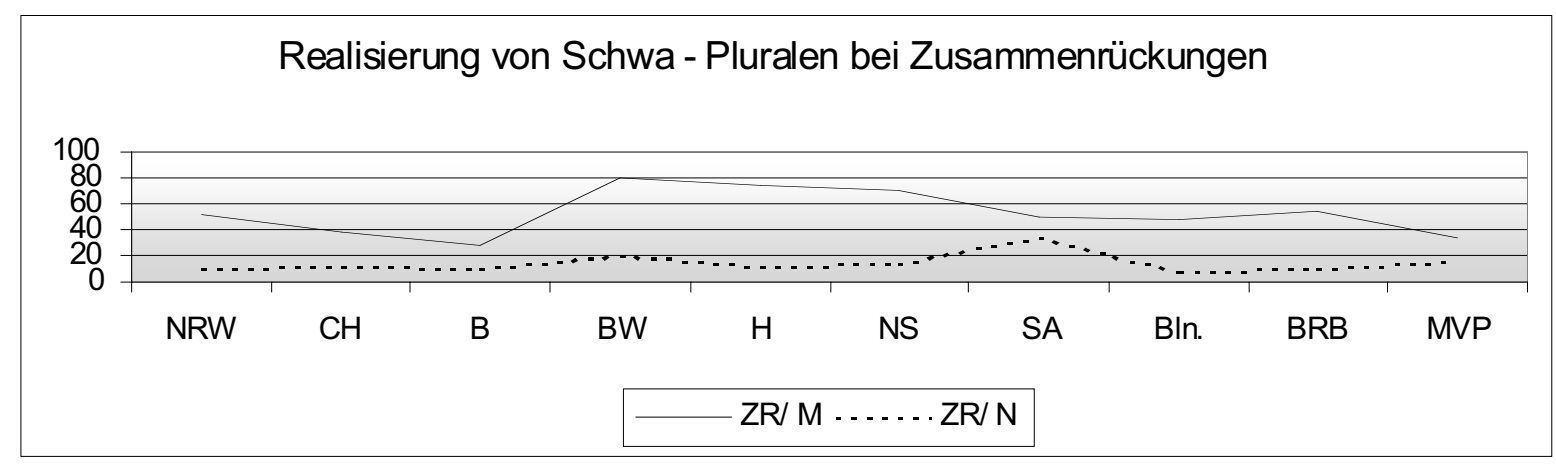

Die höheren Werte für den $e$-Plural beim Maskulinum Tunichtgut im Vergleich zum Neutrum Vergissmeinnicht sind schwer zu erklären. Sie können nicht durch das Alter der Wortformen erklärt werden, denn für Tunichtgut gibt Mackensen o.J. das 17. Jahrhundert, für Vergissmeinnicht das 15. Jahrhundert an, ebenso Kluge 1995. Prosodisch führt der $e$-Plural in beiden Fällen zum Trochäus: 'Tunicht'gute, Ver'gissmein `nichte. Der häufigere $s$-Plural bei letzterem, also Vergissmeinnichts, könnte darauf beruhen, dass beide Varianten in ihrem Letztglied homonym mit existierenden Wörtern sind: nichts und Nichte, und dass die Assoziation mit ersterem nahe liegt, während sie mit letzterem vermieden werden soll. Assimilation kann komplex sein! 